\title{
Group-wise Construction of Reduced Models for Understanding and Characterization of Pulmonary Blood Flows from Medical Images
}

\author{
Romain Guibert ${ }^{\mathrm{b}, \mathrm{a}}$, Kristin McLeod ${ }^{\mathrm{c}, \mathrm{a}}$, Alfonso Caiazzo ${ }^{\mathrm{e}}$, Tommaso Mansi ${ }^{\mathrm{f}}$, Miguel A. \\ Fernández $^{\mathrm{b}}$, Maxime Sermesant ${ }^{\mathrm{c}}$, Xavier Pennec ${ }^{\mathrm{c}}$, Irene E. Vignon-Clementel ${ }^{\mathrm{b}}$, Younes \\ Boudjemline $^{\mathrm{d}}$, Jean-Frédéric Gerbeau ${ }^{\mathrm{b}}$ \\ ${ }^{a}$ Joint first authors \\ ${ }^{b}$ Inria Paris Rocquencourt, ReO Project, Rocquencourt, France \\ ${ }^{c}$ Inria Sophia Antipolis - Méditerranée, AsclePios Project, Sophia Antipolis, France \\ ${ }^{d}$ Service de Cardiologie Pédiatrique, Hôpital Necker-Enfants Malades, Paris, France \\ ${ }^{e}$ WIAS Berlin, Berlin, Germany \\ ${ }^{f}$ Imaging and Computer Vision, Siemens Corporate Research and Technology, Princeton, NJ USA.
}

\begin{abstract}
3D computational fluid dynamics (CFD) in patient-specific geometries provides complementary insights to clinical imaging, to better understand how heart disease, and the side effects of treating heart disease, affect and are affected by hemodynamics. This information can be useful in treatment planning for designing artificial devices that are subject to stress and pressure from blood flow. Yet, these simulations remain relatively costly within a clinical context. The aim of this work is to reduce the complexity of patient-specific simulations by combining image analysis, computational fluid dynamics and model order reduction techniques. The proposed method makes use of a reference geometry estimated as an average of the population, within an efficient statistical framework based on the currents representation of shapes. Snapshots of blood flow simulations performed in the reference geometry are used to build a POD (Proper Orthogonal Decomposition) basis, which can then be mapped on new patients to perform reduced order blood flow simulations with patient specific boundary conditions. This approach is applied to a data-set of 17 tetralogy of Fallot patients to simulate blood flow through the pulmonary artery under normal (healthy or synthetic valves with almost no backflow) and pathological (leaky or absent valve with backflow) conditions to better understand the impact of regurgitated blood on pressure and velocity at the outflow tracts. The model reduction approach is further tested by performing patient simulations under exercise and varying degrees of pathophysiological conditions based on reduction of reference solutions (rest and medium backflow conditions respectively).
\end{abstract}

Keywords: Computational Fluid Dynamics, pulmonary artery, Tetralogy of Fallot, model order 
reduction, proper orthogonal decomposition, atlas construction, exercise and pathophysiological conditions

\section{Introduction}

Congenital heart disease (CHD) is a prominent cause of infant mortality around the world and decreases the quality of living and life expectancy of many. Abnormal heart rhythm and structure affect blood flow through the heart and into the arteries, which consequently influences the development of the organs and surrounding structures. CHD patients are monitored with several imaging modalities, which are chosen in medical practice based on the pathology and its severity (Puranik et al., 2010). Anatomy can be easily visualized in the images extracted from typical cardiac imaging modalities such as cine-MR in magnetic resonance (MR) imaging, echocardiography and CT. However current methods for imaging blood flow in a three-dimensional volume are limited. Non-invasive imaging techniques such as phase contrast magnetic resonance imaging (PC-MRI) are used to quantitatively measure blood flow in clinical practice only if necessary, and are not easy to acquire at multiple locations, especially for CHD patients (Markl et al., 2012). Compared to PC-MRI, cardiac MR volumetry has been shown to provide non-interchangeable cardiac function assessment in dobutamine stress testing of regurgitant repaired Tetralogy of Fallot (ToF) (Valverde et al., 2011). Time-resolved 3D contrast-enhanced MR angiography has also been proposed to non-invasively assess lung perfusion in ToF (Tomasian et al., 2009). 4D-MRI, while potentially providing velocity in a three-dimensional volume over time, is still a subject of research for its acquisition and post-processing (Markl et al., 2012). Velocity can be measured with Doppler ultrasound machines, which are more often found in clinical practice, but the acquired data is limited to $1 \mathrm{D}$.

This is one reason why computational simulations of blood flow have been developed in recent years in an attempt to better understand blood flow dynamics. The aim of such modeling is to gain insight into how hemodynamics change due to a pathology (see, e.g., (Yeung et al., 2006; Troianowski et al., 2011; LaDisa et al., 2011)). Conversely, pathology developments can depend on abnormal hemodynamics, such as in the absence of a functioning valve, as will be the case in this work. Computer hemodynamic simulations provide a tool to predict hemodynamic changes due to surgical repair (Vignon-Clementel et al., 2010b), explore different scenarios for treatment (see, e.g., (Hsia et al., 2011; Yang et al., 2011)), non-invasively compute indices that are otherwise 
invasively measured such as fractional flow reserve (FFR) (Koo et al., 2011), and design artificial devices or conduits that are subject to blood flow (see, e.g., (Prasad et al., 2011; Pant et al., 2011)). Similarly, knowing the anatomy and hemodynamics of a patient, physicians can determine the suitability of a patient for surgical intervention and plan therapy (Morales et al., 2011).

Computational fluid dynamics (CFD) methods are commonly used to simulate blood flow among others in the larger arteries, by numerically solving the three-dimensional Navier-Stokes equations. However, the large computational cost of numerical simulations is still a relevant issue, especially when dealing with patient-specific geometries. On one hand, the computation time can be reduced by exploiting parallel computing and GPU implementations (see, e.g., (Biswas, 2010)). It can also be drastically reduced when lower-dimensional models (i.e. 1D wave propagation or 0D lumped models) can efficiently replace 3D models (Moore et al., 2005; Blanco et al., 2009; Grinberg et al., 2011; Formaggia et al.; Reymond et al., 2012). However, 3D models are really warranted when pressure losses and velocity features are largely determined by the interplay between hemodynamics and complex geometry Keshavarz-Motamed et al. (2012); Itu et al. (2013), as in these repaired ToF cases. On the other hand, the issue of complexity has also been addressed by developing so-called model order reduction techniques, which aim to reduce the dimension of the problem by restricting the numerical solution to a pre-defined low order space.

Among others, in (Manzoni et al., 2011), the authors propose to extract a low dimensional parametrization of the computational domain and solve the parameterized flow equations with the reduced basis method. This work is able to gain speed in computational time but necessitates a shape parametrization of the object through control points assigned at regions of shape change. Here, we consider a model reduction based on the Proper Orthogonal Decomposition (POD, also known as Karhunen - Loève expansion or Principal Component Analysis - PCA), an approach which aims to find a low-order basis for the numerical solution starting from a set of pre-computed snapshots. In fluid dynamics, POD was introduced by Lumley in the late 1960s to study turbulent flows. For a more recent presentation of POD, we refer for example to (Volkwein, 1999; Bergmann et al., 2009; Rathinam and Petzold, 2004). In the context of blood flows, POD was used by (Mcgregor et al., 2008; McGregor et al., 2009), to interpolate flow field measured on medical images.

In this paper, we construct reduced order models of blood flows through the pulmonary artery by transporting a POD basis from a reference geometry onto patient specific anatomies. The 
reference geometry is obtained by computing a centered atlas of a preliminary population, with a non-parametric method which is thus not dependent on a choice of control points. The general methodology was briefly presented in (McLeod et al., 2010) where we only assessed the capability of the reduced basis to approximate the solution of the full order model. In the present work, we extensively test our approach on a significant number of patients, varying not only the geometry but also the experimental conditions, and we actually use the reduced order model to solve the Navier-Stokes equations.

We apply the method to a data-set of 17 pulmonary arteries (PA) with repaired ToF (as shown in Fig. 1), where the structures are affected by a known shape abnormality due to both the pathology and the initial surgical repair. A schematic diagram showing the pipeline of this method is shown in Fig 2. For this pathology, clinicians are interested in understanding how the artery remodels due to

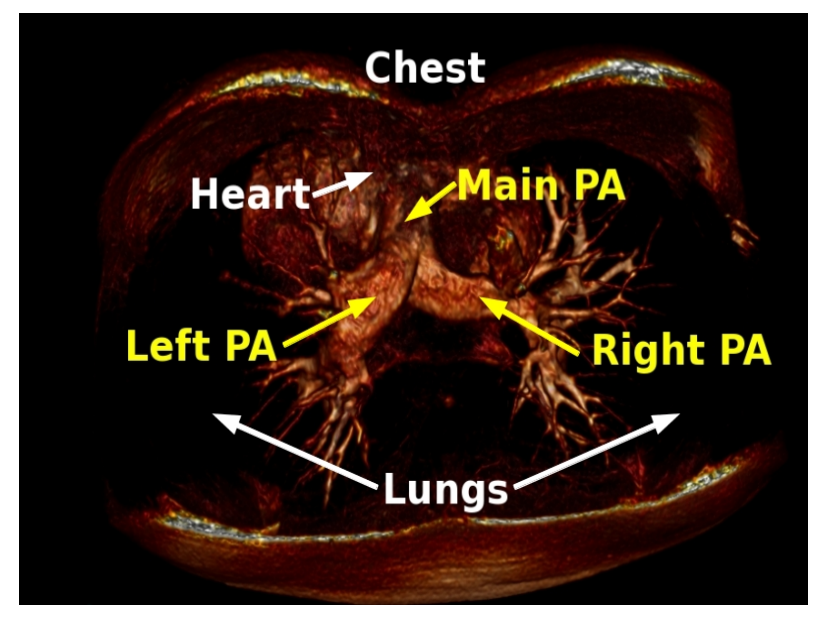

Figure 1: A 3D volume rendered image of one patient to visualise the location of the pulmonary artery (PA) with respect to the heart and lungs.

blood regurgitating back into the right ventricle of the heart, and reversely how blood regurgitates due to the deformed artery. Regurgitation results from the absence of a functioning pulmonary valve that maintains one-way blood flow from the right ventricle to the pulmonary artery. Previous ToF blood flow simulations have investigated regurgitation with lumped (Kilner et al., 2009) or idealized (geometry and boundary conditions) three-dimensional (Chern et al., 2008) models, as well as pressure losses for two repair options with either a one-dimensional (Spilker et al., 2007) or a steady three-dimensional (Chai et al., 2010) patient-specific model. In this paper, we combined 
realistic models of form (three-dimensional geometries) and function (physiological inflow and outlet boundary conditions) as in (Das et al., 2011). This is particularly important for one of the aims of this paper: to test the robustness of model order reduction not only with respect to geometry deformation, but also against changes in the physiological boundary condition. Specifically, we investigated how the reduced method works for normal as well as pathological flows in the PAs, and its capability for simulating other physiological states, namely exercise and varying degrees of pathological regurgitation. This aspect has to our knowledge not been investigated before.

For follow-up treatment planning, clinicians are interested in determining first the optimal method of intervention for each patient, be it surgical or non-invasive, as well as designing artificial devices to act in the same way as the pulmonary valve to enforce one way blood flow. Due to the complex and remodeled pulmonary artery, it is not always possible to insert existing devices in some patients. This work constitutes thus a first step towards the use of atlas-based reduced models to better understand hemodynamics in these patients and efficiently simulate hemodynamics for later device design.

The paper is structured as follows. In Sec. 2 the construction of the atlas which serves as the reference geometry is described. Sec. 3 is dedicated to blood flow simulations, describing the numerical method for the full simulations on the reference geometries and the model order reduction technique for the patient-specific reduced simulations. The procedure is assessed in Sec. 4 by comparing the reduced method to full CFD simulations in different clinically relevant situations. Finally, in Sec. 5 the results and future paths for improvements are discussed, while. Sec. 6 draws a few concluding remarks.

\section{Computation of an average geometry}

Let us assume to have a set of patient geometries drawn from a given population, and, for each patient, a surface representation of a region of interest (such as an organ, or artery), which is defined by delineating the boundaries of the organ on the images. As a first step, given the set of such surfaces we would like to compute an atlas, i.e. an average surface representation of the population. In this case, the geometry of the atlas should be well-defined and centered with respect to the population. For this we use the framework proposed in (Durrleman et al., 2009), which is described briefly in Sec. 2.1 - 2.3. Additionally, we also require a consistent method for computing individual maps from the original patient geometries to the atlas. We extend on the mathematical 


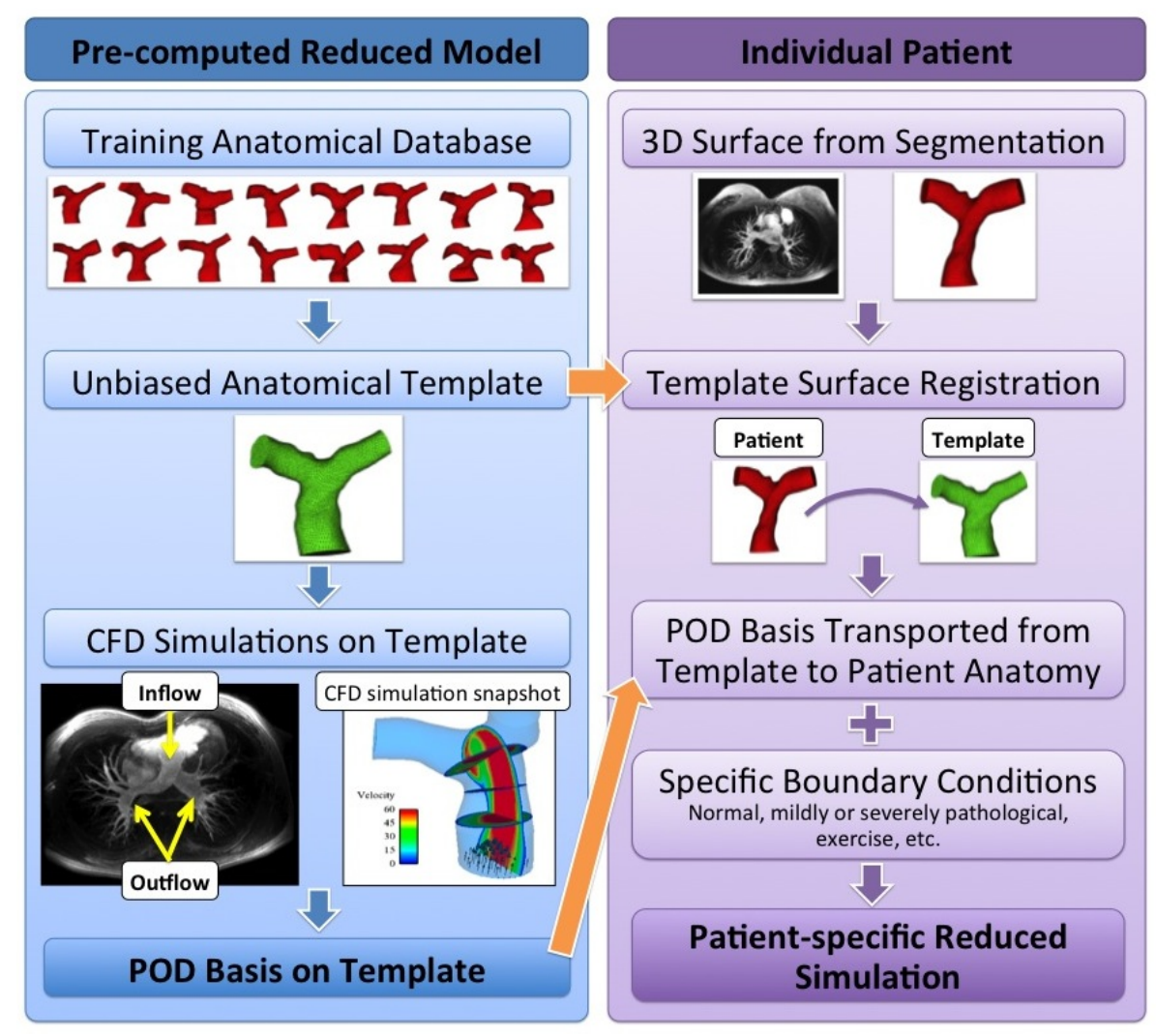

Figure 2: Schematic diagram to display the method which consists of an offline pre-computation step by simulating the blood flow on a reference geometry, and then reducing the output of the simulation to obtain a reduced-order basis. An individual flow simulation can be done by transporting the reduced-order reference basis to the individual, and solving the flow equations with the reduced basis in the patient space with patient-specific boundary conditions.

framework of (Durrleman et al., 2009) to estimate a surface representation of the centered currents atlas, as described in Sec. 2.4.

\subsection{Non-parametric representation of surfaces using currents}

As in (Durrleman et al., 2009), the patient meshes are represented by currents from the geometric integration theory. The basic idea is to characterize shapes by the flux of any vector field through them, somehow in the way 3D objects are probed by laser scanners from many locations to reconstruct their surfaces. Formally, current are elements of the metric dual of a Hilbert space of vector fields (the kernel trick from machine learning is used to provide a convenient metric), which 
gives a nice linear embedding space for anatomical surfaces. Currents are used to represent the observations (the patient geometries), the residuals (what is not captured by the geometrical model), and the deformations (used to map one geometry to another), in the same common framework. More specifically, the space of currents forms a vector space, and we can compute the distance between two meshes in the space of currents, without requiring a point-to-point correspondence between the meshes (Durrleman et al., 2009). Moreover, we can apply statistical operations such as the mean and variance on surfaces. The approach is summarized in more details in the Appendix (Sec. 7), while we refer the reader to (Durrleman et al., 2009) for a complete derivation.

\subsection{Surface-to-surface registration}

After having represented the meshes as currents, we need a method for computing the deformations, i.e. the transformations from one geometry to another. We would like to restrict the transformations to those which preserve the topology of the object and give a one-to-one (invertible) smooth transformation (i.e. a diffeomorphism). Restricting to diffeomorphisms gives non-linear deformations that allow local smooth variations to be captured in the registration. We use a group of diffeomorphisms to allow computations with discrete parametrization using the Large Deformation Diffeomorphic Metric Mapping (LDDMM) method. LDDMM was used for instance in (Beg et al., 2004 ) to find the deformations between full 3D images in the context of cardiac anatomy. Our choice is dictated by the ability of LDDMM to capture the large shape variability observed in the population. In particular, this method allows to estimate the optimal deformation $(\phi)$ from one surface to another. This deformation framework can also be used to register surfaces modeled as currents, as shown in (Vaillant and Glaunes, 2005). A brief overview of the LDDMM method is given in the Appendix (Sec. 7).

\subsection{Iterative estimation of the atlas}

Let us denote with $S_{i}, i=1, \ldots, N_{P}$ the set of patient surfaces. An atlas surface $\widehat{S}$ can be computed using a forward approach (Allassonnière et al., 2006) by modeling the observations (the patient meshes) as noisy deformations of the atlas:

$$
S_{i}=\phi_{i}(\widehat{S})+\epsilon_{i}
$$

In the latter, $\epsilon_{i}$ is a residual term that accounts for shape features not represented by the atlas. The deformations $\phi_{i}$ can be computed iteratively by minimizing the distance from the $S_{i}$ 's to the 
atlas, in order to center the atlas with respect to the observations (see Appendix). This approach is clearly disentangling the shape changes (encoded in the deformation) from the measurement noise (encoded in the residuals). Thus, statistical analyses can be done on the deformations and the residuals.

The iterative procedure can be summarized as follows (see Algorithm 1). The atlas is first initialized by taking the mean of the patient meshes in the space of currents. At each iteration $N$, the current atlas $\widehat{S}^{N}$ is then registered to each of the patients, computing individual deformations $\phi_{i}^{N}$. The new atlas is computed through a deformation, in order to minimize the error, for each patient, between the patient mesh $S_{i}$ and the geometry generated from mapping the atlas onto the patient mesh. Formally, denoting with $\|$.$\| the L_{2}$ norm (in the space of currents), we minimize the error:

$$
\varepsilon\left(\widehat{S}^{N+1}\right)=\left\|S_{i}-\phi_{i}^{N}\left(\widehat{S}^{N}\right)\right\|^{2}
$$

(see (Durrleman et al., 2009) for details on the minimization strategy). We then register the updated atlas to the individuals, recompute the atlas and loop until convergence using the forward model.

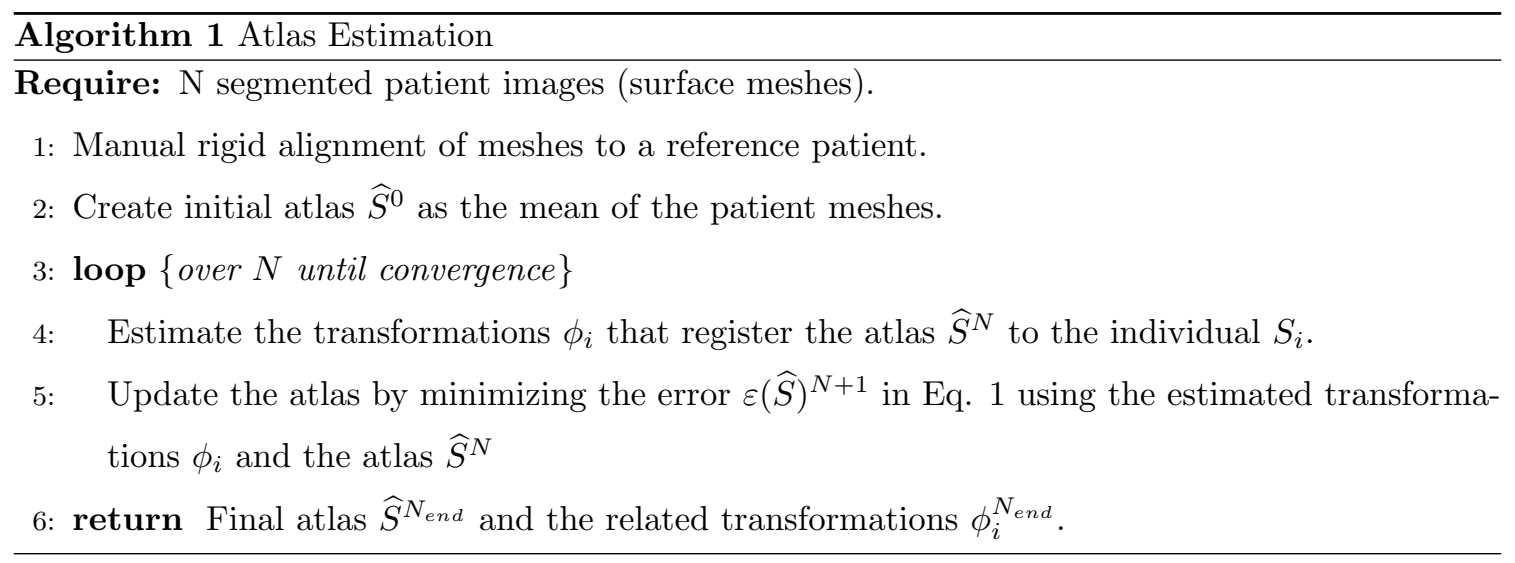

\subsection{Surface representation of the atlas}

Using the atlas-construction method described in Algorithm 1, we compute an atlas in the space of currents which can be visualized as the disjoint set of triangles (Dirac currents). This representation is sufficient for computing statistics between objects in the space of currents, however in this case we need a surface representation of the atlas in which to compute the "average" flow. A general practice for computing this surface is to register the closest patient (in the space of 
currents) to the computed atlas Of course, this representation is biased by which patient is used. To reduce this bias, we followed the minimization strategy as in (Durrleman et al., 2008) at a coarse scale, followed by an additional step to average the atlas-to-patient deformations. This average deformation was applied to the closest patient and this mesh was used to initialize the atlas construction pipeline at a finer scale. The initial optimization (Algorithm 1) is performed at a coarse scale to extract the regional differences in order to obtain a reasonable first estimate of the atlas, which is then refined to capture smaller local shape features (see Algorithm 2).

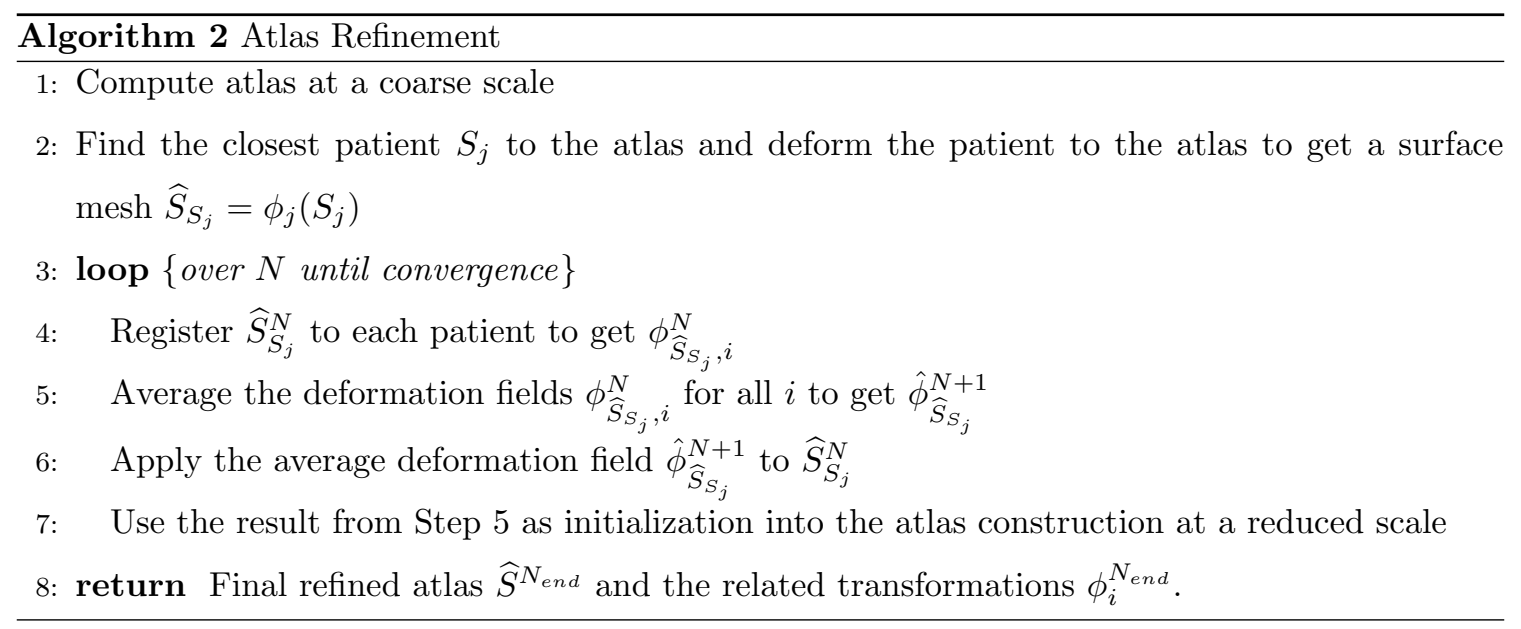

\section{An atlas-based reduced order model of blood flow}

\subsection{Reference blood flow simulation and POD basis}

In the following, let us denote by $\widehat{\Omega}$ the three-dimensional spatial domain enclosed by the atlas surface representation (Section 2.4). We will refer to $\widehat{\Omega}$ as the reference geometry. In the context of the computational fluid dynamics (CFD) in the pulmonary artery, we assume that the boundary of $\widehat{\Omega}$ is partitioned as

$$
\partial \widehat{\Omega}=\Sigma_{\text {in }} \cup \Sigma_{\text {wall }} \cup \Sigma_{\text {out }}
$$

which corresponds to the inflow, arterial wall and the outflow boundaries, respectively.

The reference simulation of blood flow is obtained by numerically solving the incompressible 
Navier-Stokes equations in $\widehat{\Omega}$, for the velocity $\hat{\mathbf{u}}: \widehat{\Omega} \times \mathbb{R}^{+} \rightarrow \mathbb{R}^{d}$ and the pressure $p: \widehat{\Omega} \times \mathbb{R}^{+} \rightarrow \mathbb{R}$

$$
\left\{\begin{aligned}
\rho \partial_{t} \hat{\mathbf{u}}+\rho \hat{\mathbf{u}} \cdot \nabla \hat{\mathbf{u}}+\nabla \hat{p}-2 \mu \operatorname{div} \boldsymbol{\epsilon}(\hat{\mathbf{u}})=0 & \text { in } \quad \widehat{\Omega}, \\
\operatorname{div} \hat{\mathbf{u}}=0 & \text { in } \quad \widehat{\Omega}, \\
\hat{\mathbf{u}}=\mathbf{u}_{\text {in }} & \text { on } \quad \Sigma_{\text {in }}, \\
\hat{\mathbf{u}}=0 & \text { on } \quad \Sigma_{\text {wall }}, \\
\boldsymbol{\sigma}(\hat{\mathbf{u}}, \hat{p}) \mathbf{n}=-p_{\text {out }} \mathbf{n} & \text { on } \quad \Sigma_{\text {out }} .
\end{aligned}\right.
$$

In (2), $\rho$ stands for the density of the fluid and the fluid Cauchy-stress tensor is given by

$$
\boldsymbol{\sigma}(\mathbf{u}, p)=-p \mathbf{I}+2 \mu \epsilon(\mathbf{u}), \quad \boldsymbol{\epsilon}(\mathbf{u}) \stackrel{\text { def }}{=} \frac{1}{2}\left(\nabla \mathbf{u}+\nabla \mathbf{u}^{\mathrm{T}}\right),
$$

$\mu$ being the dynamic viscosity of the fluid, $\mathbf{u}_{\text {in }}$ a given inlet velocity field and $p_{\text {out }}$ an outlet pressure (defined below).

At the inlet we prescribe a parabolic velocity profile, whose flow rate varies in time according to a chosen physiological regime. At the outlet, a relationship between pressure and flow is prescribed, in order to represent the pulmonary vessels downstream of the 3D-fluid model. We consider an outlet boundary composed of two disjointed surfaces, i.e. $\Sigma_{\text {out }}=\Gamma_{1} \cup \Gamma_{2}$. The outlet pressure $p_{\text {out }}$ at each outlet boundary $\Gamma_{i} \subset \Sigma_{\text {out }}$ is defined by a lumped parameter model, in which $p_{\text {out }}(t)$ is related to the outgoing flux at $\Gamma_{i}$,

$$
Q_{\mathrm{out}}^{i}(t)=\int_{\Gamma_{i}} \mathbf{u}(t) \cdot \mathbf{n} d s
$$

in an analogous way as voltage and current are related in electric circuits. In particular, we used a 3-element Windkessel model (Frank, 1899) (see (Vignon-Clementel et al., 2010a) for recent applications in pulmonary artery modeling), in which the outlet pressure and flow are related by the ordinary differential equation:

$$
p_{\text {out }}+R_{\mathrm{d}} C \frac{\mathrm{d} p_{\text {out }}}{\mathrm{d} t}=\left(R_{\mathrm{p}}+R_{\mathrm{d}}\right) Q_{\text {out }}+R_{\mathrm{p}} R_{\mathrm{d}} C \frac{\mathrm{d} Q_{\text {out }}}{\mathrm{d} t},
$$

where $R_{\mathrm{p}}$ and $R_{\mathrm{d}}$ model the resistance of the proximal and distal vasculature, respectively, and the capacity $C$ takes into account the ability of the downstream vessels to store blood during peak flow and recoil when pressure decreases. The deformability of the vessels could also been taken into account in the 3D domain: fluid-solid interaction has been shown to influence both pressure and flow in pulmonary arteries, changing not so much the streamlines but more the pressure and wall 
shear stress, mostly for exercise conditions (Bazilevs et al., 2009). However this requires knowledge of thickness and material properties of the wall, which cannot be easily extracted from the available MRI data. As a first step the wall is thus assumed rigid, although the proposed method could be also extended to the case of fluid-solid interaction.

\subsubsection{Numerical approximation}

Problem (2) is discretized in time with a Chorin-Temam projection scheme (see, e.g., (Guermond et al., 2006; Chorin, 1968; Temam, 1968)) in which velocity and pressure are solved separately in

two substeps. Namely, let us denote with $\tau$ the time-step size, setting $t_{n} \stackrel{\text { def }}{=} n \tau$ for $1 \leq n \leq N$. For a given initial condition $\mathbf{u}^{0}=\mathbf{u}_{0}$, the time iteration consists of solving the two problems:

1. Velocity step:

$$
\left\{\begin{array}{rcc}
\rho \frac{\hat{\mathbf{u}}^{n+1}-\hat{\mathbf{u}}^{n}}{\tau}+\rho \hat{\mathbf{u}}^{n} \cdot \nabla \hat{\mathbf{u}}^{n+1}-2 \mu \boldsymbol{\nabla} \cdot \boldsymbol{\epsilon}\left(\hat{\mathbf{u}}^{n+1}\right)+\nabla \hat{p}^{n}=\mathbf{0} & \text { in } \quad \widehat{\Omega} \\
\hat{\mathbf{u}}^{n}=\mathbf{u}_{\mathrm{in}}\left(t_{n+1}\right) & \text { on } \quad \Sigma_{\text {in }}, \\
2 \mu \epsilon\left(\hat{\mathbf{u}}^{n+1}\right) \mathbf{n}=\mathbf{0} & \text { on } \quad \Sigma_{\text {out }}, \\
\hat{\mathbf{u}}^{n}=\mathbf{0} & \text { on } \quad \Sigma_{\text {wall }} .
\end{array}\right.
$$

2. Pressure-Poisson projection step:

$$
\left\{\begin{aligned}
-\frac{\tau}{\rho} \Delta \hat{p}^{n+1} & =-\boldsymbol{\nabla} \cdot \hat{\mathbf{u}}^{n+1} \quad \text { in } \quad \widehat{\Omega} \\
& \frac{\tau}{\rho} \frac{\partial \hat{p}^{n+1}}{\partial \mathbf{n}}=0 \quad \text { on } \quad \Sigma_{\text {in }} \cup \Sigma_{\text {wall }} \\
\hat{p}^{n+1}=p_{\text {out }}^{n+1} & \text { on } \quad \Sigma_{\text {out }}
\end{aligned}\right.
$$

The 3D-0D coupling on the outlet boundary condition is treated in a implicit fashion (see (Bertoglio et al., 2013)). The discretization in space of problems (5)-(6) is performed via continuous piecewise affine finite elements. We denote by $\boldsymbol{V}_{h}$ and $Q_{h}$ the corresponding finite element spaces for the velocity and the pressure, respectively.

\subsubsection{Proper orthogonal decomposition on the reference geometry}

A proper orthogonal decomposition (POD) of a numerical solution (that is $\left\{\hat{\mathbf{u}}_{h}^{n}\right\}_{i=1}^{N}$ and $\left\{\hat{p}_{h}^{n}\right\}_{n=1}^{N}$ or, in general, of a given set of data, see (Bergmann et al., 2009; Rathinam and Petzold, 2004) for instance), consists of finding a set of basis functions (orthogonal w.r.t. a given scalar product) 
which, even containing a small number of elements, can represent sufficiently well the numerical solution. This approach, besides reducing the data size without losing relevant information, allows to perform faster numerical simulations, by restricting the space of the solution to the subspace generated by the POD basis functions.

Starting from a full CFD simulation on the reference geometry, we computed two POD bases for velocity and for pressure, denoted by $\hat{\boldsymbol{\Phi}}=\left\{\hat{\boldsymbol{\varphi}}_{i}\right\}_{i=1}^{M_{\mathbf{u}}}$ and $\hat{\Psi}=\left\{\hat{\psi}_{i}\right\}_{i=1}^{M_{p}}$ respectively. Note that with $\hat{\varphi}_{i}: \widehat{\Omega} \rightarrow \mathbb{R}^{3}$ and $\hat{\Psi}_{i}: \widehat{\Omega} \rightarrow \mathbb{R}$, these basis functions are given in terms of their natural decomposition on the finite element basis of $\boldsymbol{V}_{h}$ and $Q_{h}$, respectively. Note that in practice we have $M_{\mathbf{u}} \ll \operatorname{dim}\left(\boldsymbol{V}_{h}\right)$ and $M_{p} \ll \operatorname{dim}\left(Q_{h}\right)$. Hence, the main idea of model reduction is to perform the spatial approximation of (5) and (6) on the lower dimensional spaces spanned by $\hat{\boldsymbol{\Phi}}$ and $\hat{\Psi}$, respectively.

For instance, using the velocity POD basis $\hat{\boldsymbol{\Phi}}$, instead of the original finite element basis of $\boldsymbol{V}_{h}$, the discrete problem (5) can be formulated in terms of the reduced solution

$$
\tilde{\mathbf{u}}(\mathbf{x}, t)=\sum_{i=1}^{M_{\mathrm{u}}} \alpha_{i}(t) \hat{\boldsymbol{\varphi}}_{i}(\mathbf{x}),
$$

requiring, at each time step, the solution of a linear system of reduced size $M_{\mathbf{u}} \times M_{\mathbf{u}}$.

\subsection{Reduced order blood flow simulation on individual patients}

To obtain the POD basis functions for pressure and velocity on the reference geometry we performed a full CFD simulation. The key idea of our method is to map these reference basis functions onto individual patient meshes, in order to obtain reduced bases without the need of full individual simulations.

The procedure is summarized in Algorithm 3. Firstly, we use the atlas geometry as a reference domain, introducing geometrical mappings from each 3D-patient mesh onto the atlas geometry (3D-shape registration, section 3.2.1). Next, we use these mappings to transport the template POD basis onto each individual domain (section 3.2.2). Finally, this allows us to perform the POD reduction of the FE formulation for the patient-specific problem (section 3.2.3).

\subsubsection{D-shape registration}

The first step towards the construction of individual POD bases is the computation of a map between the reference and each individual mesh. In particular, let $\hat{\Omega}$ and $\Omega$ denote the spatial 
domain of the reference and patient geometries, respectively, and let us consider the discretized representations of $\hat{\Omega}$ and $\Omega$, defined by two tetrahedral meshes $\hat{\mathcal{T}}_{h}$ and $\mathcal{T}_{h}$. We aim to compute a map

$$
\mathcal{A}: \hat{\Omega} \rightarrow \Omega
$$

which maps $\hat{\mathfrak{T}}_{h}$ onto $\mathcal{T}_{h}$ preserving the mesh topology. In particular, $\mathcal{A}$ defines a one-to-one correspondence between the nodes of the two meshes. In the following, $\mathcal{A}$ will be called a $3 D$-shape registration.

To construct $\mathcal{A}$, we start from the surface diffeomorphism (introduced in section 2.2)

$$
\phi: \widehat{S} \rightarrow S
$$

which maps the atlas surface $\hat{S}$, i.e. the boundary of the reference domain, onto an approximation of the patient surface $S$. Since, in the discrete formulation, $\hat{S}$ and $S$ are represented by triangular surface meshes, the diffeomorphism $\phi$ is a piecewise linear deformation defined on the whole surface. Let us denote this deformation as a displacement field $\mathbf{d}_{s}: \partial \widehat{\Omega} \rightarrow \mathbb{R}^{3}$ such that

$$
\partial \Omega=\partial \widehat{\Omega}+\mathbf{d}_{s}(\partial \widehat{\Omega}) .
$$

The 3D registration $\mathcal{A}$ will be defined as an extension of the field $\mathbf{d}_{s}$ onto the three-dimensional domain with a suitable volume deformation field

$$
\mathbf{d}_{v}: \hat{\Omega} \rightarrow \mathbb{R}^{3},
$$

compatible with the surface displacement $\mathbf{d}_{s}$ on the boundary. In particular, once $\mathbf{d}_{v}$ is computed, we can define the deformation as

$$
\mathcal{A}(\hat{\mathbf{x}})=\hat{\mathbf{x}}+\mathbf{d}_{v}(\hat{\mathbf{x}}), \text { for } \hat{\mathbf{x}} \in \hat{\Omega} .
$$

There are different choices available for the computation of the extension $\mathbf{d}_{v}$. For instance, one could take the harmonic extension of $\mathbf{d}_{s}$ into $\widehat{\Omega}$. However, since the displacement field $\mathbf{d}_{v}$ will be used to deform the computational mesh, this simple extension could yield poor quality meshes, especially if the surface deformation is large.

Instead, we decomposed the boundary deformation $\mathbf{d}_{s}$ in $K$ sub-steps and computed the extension $\mathbf{d}_{v}$, by solving a sequence of harmonic problems, for $k=0, \ldots, K-1$

$$
\begin{array}{r}
\Delta \mathbf{d}_{v}^{k+1}=0, \quad \text { in } \widehat{\Omega}^{k} \\
\mathbf{d}_{v}^{k+1}=\frac{k+1}{K} \mathbf{d}_{s}, \text { on } \partial \widehat{\Omega}^{k},
\end{array}
$$


considering at each step a new computational domain, defined by $\widehat{\Omega}^{k+1}=\widehat{\Omega}^{k}+\mathbf{d}^{k}$.

This procedure corresponds to a non-linear extension of the surface displacement, obtained by solving a sequence of linear problems (11). In particular, it allows the size of the boundary deformation imposed at each sub-step to be controlled by the value of $K$. This iterative approach could be replaced by other non-linear extensions of the surface displacement, e.g. considering the volume mesh as a hyperelastic material, which might be more robust in the case of very large deformations (see a stricking example in Cairncross et al. (2000), p. 382). Notice that each solution

of (11) on the reference mesh $\hat{\mathfrak{T}}_{h}$ automatically yields a deformation which preserves the topology of the reference mesh, i.e. such that the nodes of the $\hat{\mathfrak{T}}_{h}$ are mapped onto the nodes of the individual volume mesh $\mathcal{T}_{h}$.

\subsubsection{Transporting the reference POD basis}

The map constructed in section 3.2.1 provides a coordinate change between reference and individual domains. This will now be used to compute individual POD bases for pressure and velocity on the patient geometries, starting from the reference reduced basis.

An individual POD basis for the pressure $\Psi$ can be obtained from the reference POD basis $\hat{\Psi}_{i}$ through a coordinate change, namely,

$$
\psi_{i}(\mathbf{x}) \stackrel{\text { def }}{=} \hat{\psi}_{i}(\hat{\mathbf{x}}), \quad \hat{\mathbf{x}}=\mathcal{A}^{-1}(\mathbf{x})
$$

for all $i=1, \ldots, M_{p}$.

To construct an individual POD basis for the velocity field on a given patient, one has to explicitly take into account the deformation of the domain, in order to preserve the properties of the vector field. To do this, we consider the inverse Piola transform of the basis elements of $\hat{\boldsymbol{\Phi}}$, given by

$$
\boldsymbol{\varphi}_{i}(\mathbf{x})=\boldsymbol{P}\left(\hat{\boldsymbol{\varphi}}_{i}\right)(\mathbf{x}) \stackrel{\text { def }}{=} \frac{1}{J(\hat{\mathbf{x}})} \mathbf{F}(\hat{\mathbf{x}}) \hat{\boldsymbol{\varphi}}_{i}(\hat{\mathbf{x}}), \quad \hat{\mathbf{x}}=\mathcal{A}^{-1}(\mathbf{x})
$$

for $i=1, \ldots, M_{\mathbf{u}}$. Here, $\mathbf{F}(\hat{\mathbf{x}})$ stands for the deformation gradient, i.e., $\mathbf{F}(\hat{\mathbf{x}}) \stackrel{\text { def }}{=} \nabla \mathcal{A}(\hat{\mathbf{x}})$, and $J(\hat{\mathbf{x}})=\operatorname{det} \mathbf{F}(\hat{\mathbf{x}})$ is the jacobian of $\mathbf{F}$. From the properties of the Piola transform (see, e.g., (Ciarlet, 1988)), we can infer that

$$
J(\hat{\mathbf{x}}) \operatorname{div}_{\mathbf{x}} \boldsymbol{\varphi}_{i}(\mathbf{x})=\operatorname{div}_{\hat{\mathbf{x}}} \hat{\boldsymbol{\varphi}}_{i}(\hat{\mathbf{x}}) .
$$

Hence, if the POD basis is divergence free in the reference geometry then the transformed basis has the same property in the individual geometry. Note that a similar transform was used in Løvgren 
et al. (2007) in a different context.

Considering the shape registration in the form (10), we approximate the gradient

$$
F(\hat{\mathbf{x}})=\mathbf{I}+\nabla \mathbf{d}_{v}(\hat{\mathbf{x}})
$$

by its $L^{2}$-projection on the piecewise linear finite element space. In practice, this is done by solving a linear system associated with the mass matrix, whose entries are given by $\hat{M}_{i j}=\int_{\widehat{\Omega}} \hat{\mathbf{v}}_{i} \hat{\mathbf{v}}_{j} d \widehat{\Omega}, \hat{\mathbf{v}}_{i}$ and $\hat{\mathbf{v}}_{j}$ being basis functions of the finite element space in $\hat{\Omega}$.

\subsubsection{Reduced simulation}

With a little abuse of notation, let us denote with $\Phi=\left(\varphi_{1}|\ldots| \varphi_{M_{\mathrm{u}}}\right)$ an individual POD basis in matrix form, where each column contains a basis element, obtained with the procedure described in Sections 3.2.1-3.2.2.

At each time step $t_{n}$, let us call $A^{n}$ and $\mathbf{f}^{n}$ the matrix and the right-hand side of the linear system associated to the finite element approximation of problem (5) in the patient geometry. The reduced model is given by

$$
\left(\Phi^{T} A^{n} \Phi\right) \widetilde{\mathbf{U}}=\Phi^{T} \mathbf{f}^{n}
$$

where $\widetilde{\mathbf{U}}=\left\{\alpha_{1} \ldots \alpha_{M_{\mathbf{u}}}\right\}^{T}$ defines the reduced numerical solution (7). Analogously, the reduced system for the pressure-problem (6) is built by considering the transported pressure basis $\Psi=$ $\left(\psi_{1}|\ldots| \psi_{M_{p}}\right)$.

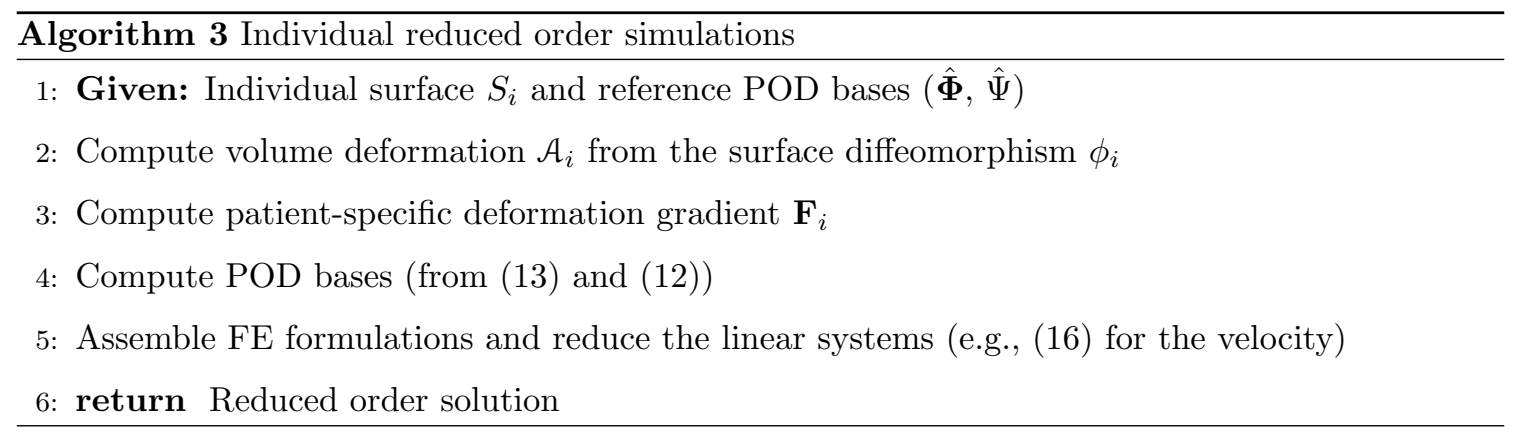




\section{Numerical experiments on the pulmonary artery of repaired tetralogy of Fallot patients}

\subsection{Data Collection}

Subjects and Image Preparation. A data-set of 17 adults with repaired tetralogy of Fallot was used in this study. MRI angiography of the heart was acquired with a $1.5 T$ scanner (Signa excite, GE Medical Systems) with isotropic in-plane resolution $0.703 \mathrm{~mm} \times 0.703 \mathrm{~mm}$ and $1 \mathrm{~mm}$ thick slices.

Image segmentation of the pulmonary artery. In order to extract the surfaces of the pulmonary artery, a user-guided 3D image segmentation tool was applied to delineate the boundaries of the artery. Using this tool, a small number of control points (less than 100) are added by the user to define the inside, outside, and border of the region. With these control points as a guide, a 3D mesh is constructed by an implicit variational surfaces approach. The tool is included within the CardioViz3D software package available for download ${ }^{1}$, see (Mansi, 2010) for further details. This tool was applied to each of the patient images to define the artery for at least $3 \mathrm{~cm}$ of the inflow before the bifurcation and at least $2 \mathrm{~cm}$ of each outflow branch after the bifurcation (see Fig. 3 ). The variable branch length in the final geometries resulted from the variability of the images used, so in the case when it was possible to include more than $2 \mathrm{~cm}$ of the outflow, this was included. In particular, images with little image information at the outflow branches resulted in a shorter segmentation.
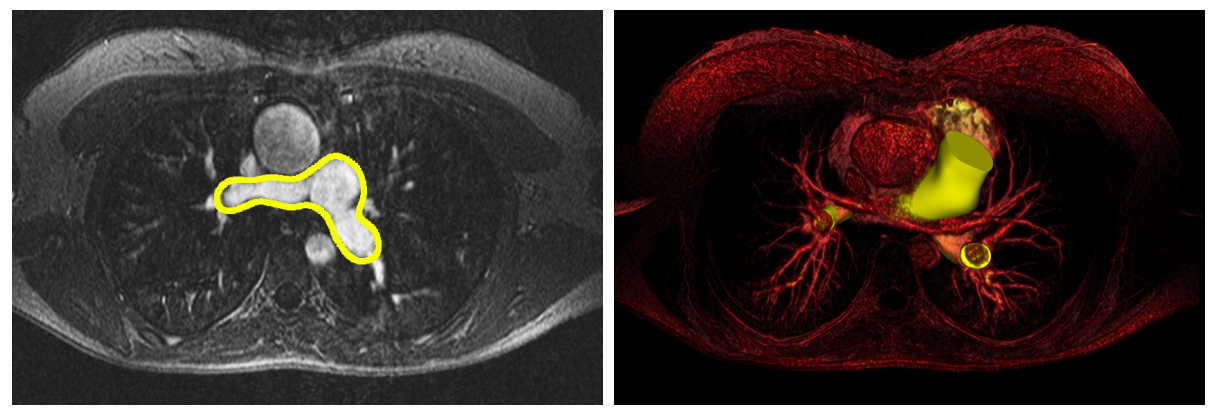

Figure 3: Left: 2D slice of one patient with the segmented outline in yellow. Right: 3D rendering of the same patient with the 3D mesh overlaid (in yellow).

\footnotetext{
${ }^{1}$ http://www-sop.inria.fr/asclepios/software/CardioViz3D/
} 


\subsection{Statistical shape model of the pulmonary arteries}

The obtained meshes were used to compute a centered atlas as a reference for the population (see Fig. 4 and 5). The atlas construction pipeline described in Sec. 2 requires two parameters to be set to control the 'stiffness' of the non-linear deformations. First, $\sigma_{V}$, the standard deviation of the LDDMM Gaussian kernel $K_{V}$, which can be seen as the typical distance of coherence of the deformation (higher values give more global transformations, such as rigid body transformations). Second, a parameter $\sigma_{W}$ (the standard deviation of the currents Gaussian kernel $K_{W}$ ), that characterizes the resolution of the currents representation, to control how finer deformations are treated as either noise or shape features. Since we were mainly interested in the regional ToF alterations related to dilation, valve enlargement, and regional bulging, these parameters were set to $\sigma_{W}=30 \mathrm{~mm}, \sigma_{V}=5 \mathrm{~mm}$ for the atlas. With the algorithm described in Sec. 2, an atlas was constructed by 4 iterations of the alternate minimization (the number of iterations needed to reach the convergence criteria). This was sufficient to give a well-centered geometry in which to apply the atlas refinement pipeline described in algorithm 2.4.

Un-biased validation of the atlas. To test how biased the atlas is to the population used to create it, we performed a leave-one-out validation by creating 17 atlases using 16 patients each. The resulting atlases are shown overlaid on one another in Fig. 6. Though there is a large shape variability observed in the population, there is little difference between each of the computed atlases.

\subsection{Patient-specific full and reduced order CFD simulations}

For the full CFD simulations on the individual geometries, rather than the original patient meshes, we used the geometries obtained by mapping the atlas to each patient via the deformation computed in the 3D-shape registration step (described in Sec. 3.2.1). Note that this results in a discrepancy between the original meshes and the atlas-to-patient deformed meshes, since we allow for some noise in the atlas construction step. However, due to the fact that the atlas is well-centered, these differences are small. The simulation approach described in Sec. 3 was applied for normal (functioning valve case) and pathological (regurgitant or absent valve) regimes. The aim was to investigate how such a reduced approach performs for both hemodynamic conditions. For each regime, a full simulation was performed on the atlas, extracting a POD basis containing 30 modes.

Then, for each of the individuals, a reduced order simulation was performed with this transported POD and the hemodynamic boundary conditions of that regime. Subsequently, for both regimes, 

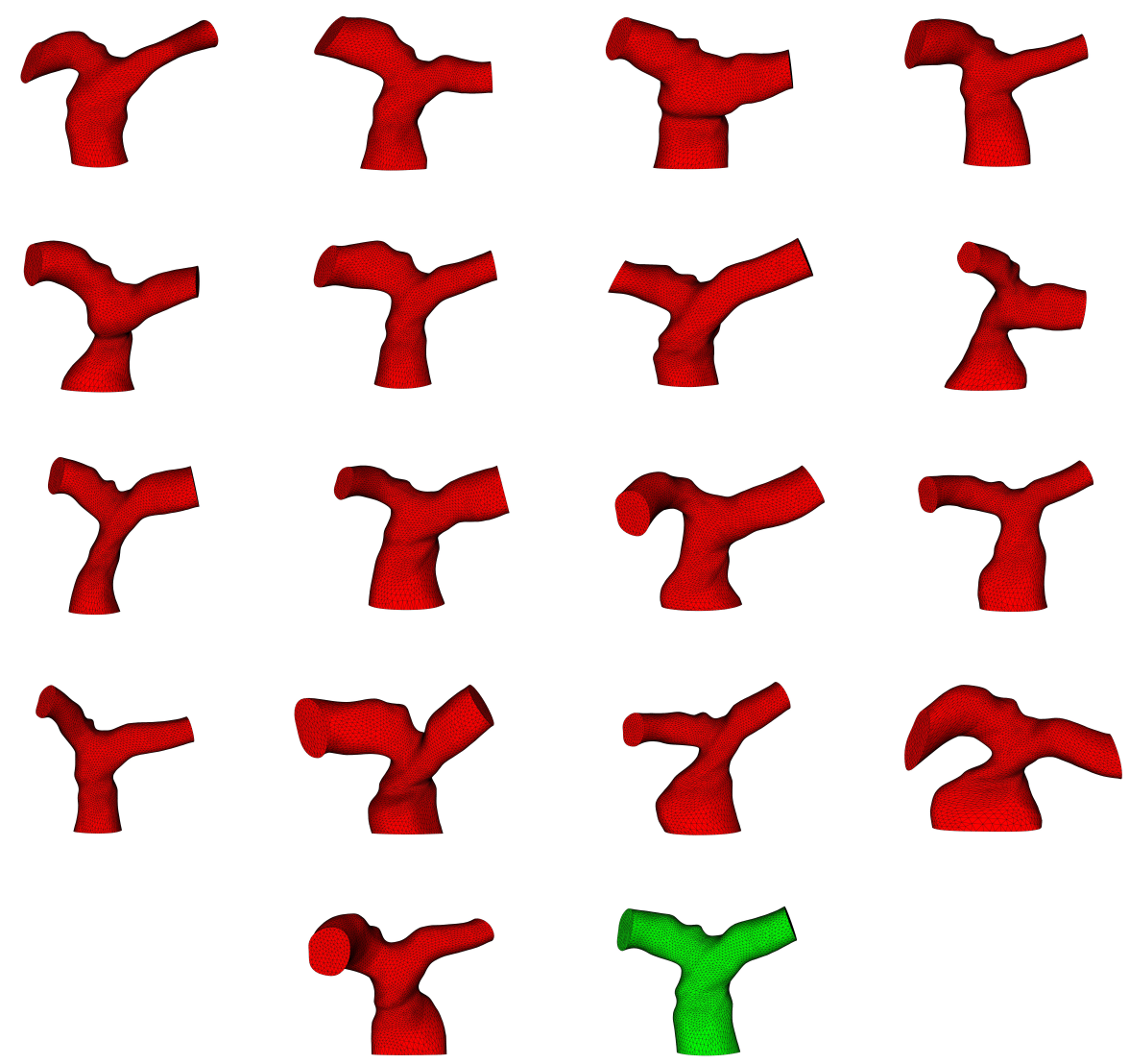

Figure 4: The 17 patient meshes (red) and associated atlas mesh (green).

the PODs built under rest conditions were tested under exercise conditions. Furthermore, the robustness of the method was assessed by investigating if the POD constructed for the reference pathological condition was able to capture other pathological conditions.

To assess the reduced order method, we simulated the flow in the different geometries both with a full FE model and with the reduced POD basis as described in Sec. 3. For each simulation, we monitored four different errors in time indicating the global errors in velocity, pressure, outlet flows through the two branches and the pressure drop between the right ventricle and the two outlets. The last two are particularly interesting from the clinical point of view, while the first two aid in assessing the accuracy of the reduced flow simulation:

- Instantaneous $L^{2}$-norm difference in velocity and pressure, adimensionalized by the maximum 

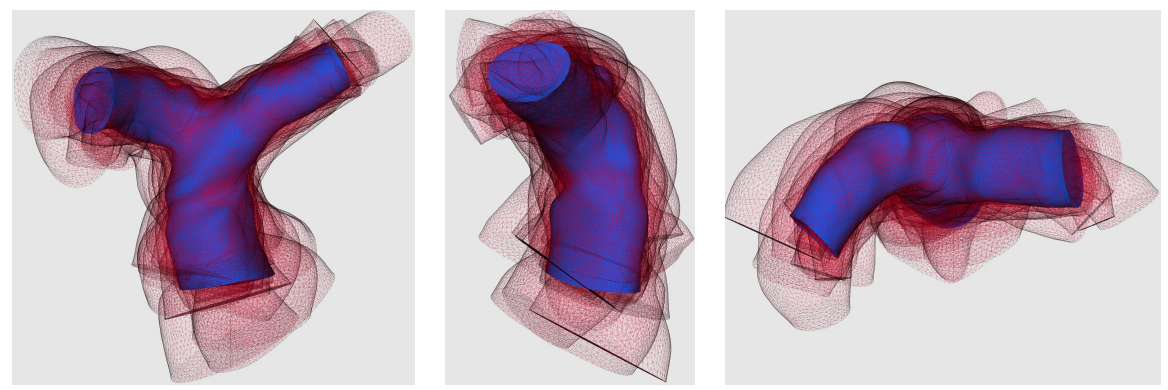

Figure 5: Front view (left), side view (center) and top view (right) of the atlas (blue) and the 17 meshes used to create the atlas (wire-frame red). Though there is a wide shape variability in the population, the atlas is well-centered.
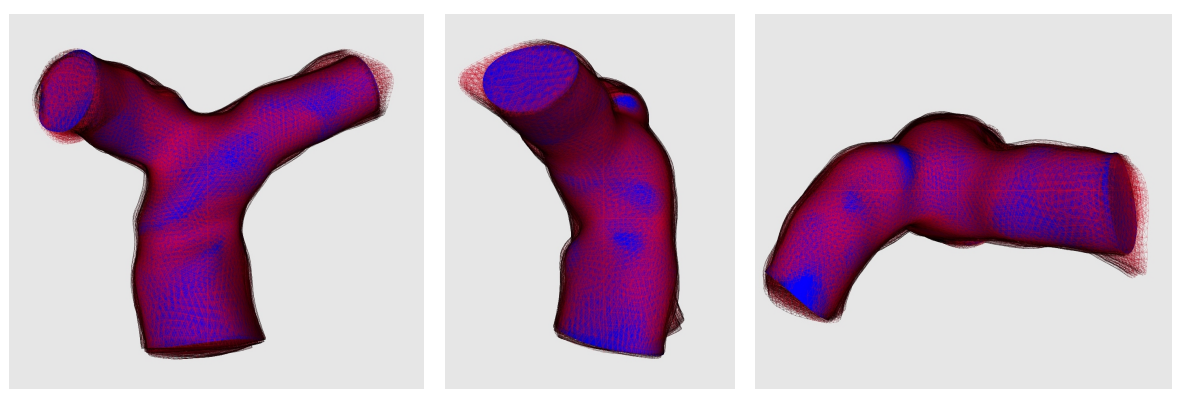

Figure 6: Front view (left), side view (center) and top view (right) of the atlas created on all the patients (blue) and the 17 atlases created on 16 patients (wire-frame red). There is little difference between the atlases, even given the high variability of shape in the population, which displays the un-biased property of the atlas construction step.

$L^{2}$-norm in time:

$$
E_{\mathbf{u}}=\frac{\left\|\mathbf{u}_{\text {reduced }}-\mathbf{u}_{\text {full }}\right\|_{L^{2}}}{\max \left(\left\|\mathbf{u}_{\text {full }}\right\|_{L^{2}}\right)} \quad \text { and } \quad E_{p}=\frac{\left\|p_{\text {reduced }}-p_{\text {full }}\right\|_{L^{2}}}{\max \left(\left\|p_{\text {full }}\right\|_{L^{2}}\right)}
$$

- Instantaneous pressure drop (difference between averaged over the surface inlet and outlet pressures), adimensionalized by the maximum value in time:

$$
E_{\Delta p}=\max _{i=1,2}\left(\frac{\left\|\left(p_{\text {in }}-p_{\text {out }}^{i}\right)_{\text {reduced }}-\left(p_{\text {in }}-p_{\text {out }}^{i}\right)_{\text {full }}\right\|}{\max \left(p_{\text {in }}-p_{\text {out }}^{i}\right)_{\text {full }}}\right)
$$

- Instantaneous outlet flow error, adimensionalized by the maximum value in time:

$$
E_{q}=\max _{i=1,2}\left(\frac{\left\|q_{\text {out reduced }}^{i}-q_{\text {out full }}^{i}\right\|}{\max \left\|q_{\text {out full }}^{i}\right\|}\right) \text {, with } q_{\text {out }}^{i}=\int_{\Gamma_{i}} \mathbf{u} \cdot \mathbf{n}
$$

Renormalizing the error w.r.t. the instantaneous norm actually leads to large and less informative relative errors when the norms of velocity and pressure are close to zero. It is more relevant 
from a practical point of view to have an idea of the error with respect to a fixed meaningful quantity. Thus the absolute errors are normalized w.r.t a constant factor which allows e.g. to compare the situation on different patients and with different boundary conditions, when peak flows and pressures might differ.

\subsubsection{POD validation: simulations on the reference geometry}
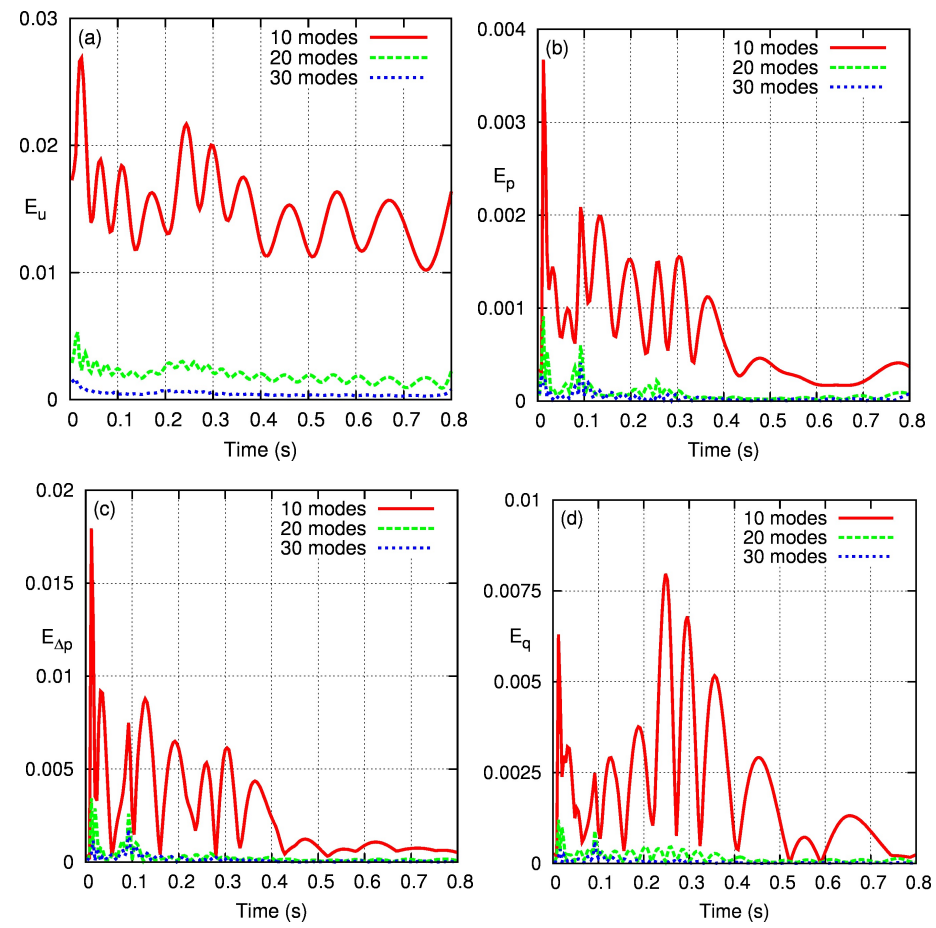

Figure 7: Comparison between full and reduced simulations on the reference geometry for (a) velocity, (b) pressure, (c) presure drop and (d) outlet flow, following (17)-(19) and variying the numbers of POD modes retained for reduced simulations.

As a preliminary validation, we tested the capability of the POD basis to solve the problem on the reference geometry only (e.g. without transporting the basis), using the inlet flow conditions depicted in figure 8 and the 0D-Windkessel model defined in table 1. Figure 7 shows the errors in time (defined in (17)-(19)) using POD bases with increasing numbers of modes for the reduced simulation. We obtained a very satisfactory accuracy with the POD basis (all errors below 1\%) using around 20 - 30 POD modes. In view of these results, all the simulations of this study were performed with 30 POD modes. Enriching the POD basis might yield a better approximation of 
the solution on the reference geometry. However, in our numerical tests, it did not reduce the errors between the full and reduced individual patient simulations, since in those cases, errors due to the transport on patient geometries were dominant.

\subsubsection{Flow simulations in normal and pathological regimes}

First, we used the pre-computed reduced models to simulate the flow in the individual geometries with different flow conditions. In particular, we distinguished between a normal cardiac flow condition, i.e. with very little backflow as it is the case in the pulmonary artery of a healthy pulmonary valve, and a pathological flow condition with $30 \%$ of backflow during diastole, the average amount of expected backflow for patients without pulmonary valves (Schwartz et al., 2011).

Note that flow curves were not available for all patients, thus the pathological flow curve corresponds to a single patient measurement, typical of such ToF physiology. The same 0D-Windkessel model was applied at both outlets, but the parameters were chosen in the simulations to obtain pressure waveforms typical of a normal pulmonary artery and right ventricle (pathological pressure in the pulmonary artery due to the absence of a valve) respectively (see table 1). The inlet flow profiles and the resulting outlet pressures for the atlas simulations for these two cases are depicted in Fig. 8 and 9. In particular, one can see the characteristic pressure bump at the end of diastole that is observed in the pulmonary artery, as in the right ventricle, for the pathological case. Furthermore, we investigated different degrees of pathological conditions, with $15 \%$ and $40 \%$ of backflow during diastole (see Fig. 9).

Remark 4.1. A POD basis transported as in (13) and (12) is associated to a single patient mesh, and can be employed to reduce a simulation for this specific geometry. It should be noted that even though the solution snapshots depend on particular essential boundary conditions, the POD basis is built from the corresponding set of snapshots with homogeneous essential boundary data, which are the genuine degrees of freedom of the system. Hence it is possible to run the reduced model with different boundary conditions, such as the inlet velocity in (5). It is also possible to vary other physical parameters, e.g. in (4). It is therefore technically possible to use the same basis for different physiological regimes. The accuracy of the reduced model has nevertheless to be tested in those cases, which is the aim of various tests presented in this paper.

The errors (17)-(18) for normal flow conditions for the different patients are shown in Fig. 10. To provide a better picture of the error variability among patients, mean errors and standard deviations 


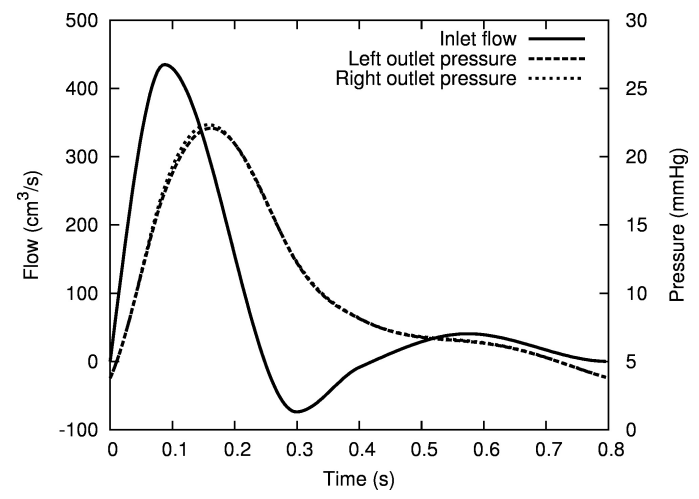

Figure 8: Normal boundary conditions: flow rate is prescribed at the inlet (with a mean value of $4.9 \mathrm{~L} \cdot \mathrm{min}^{-1}$ ) and outlet pressure for the atlas obtained the Winkessel model in Table 1. These outlet pressures change slightly from one geometry to another.
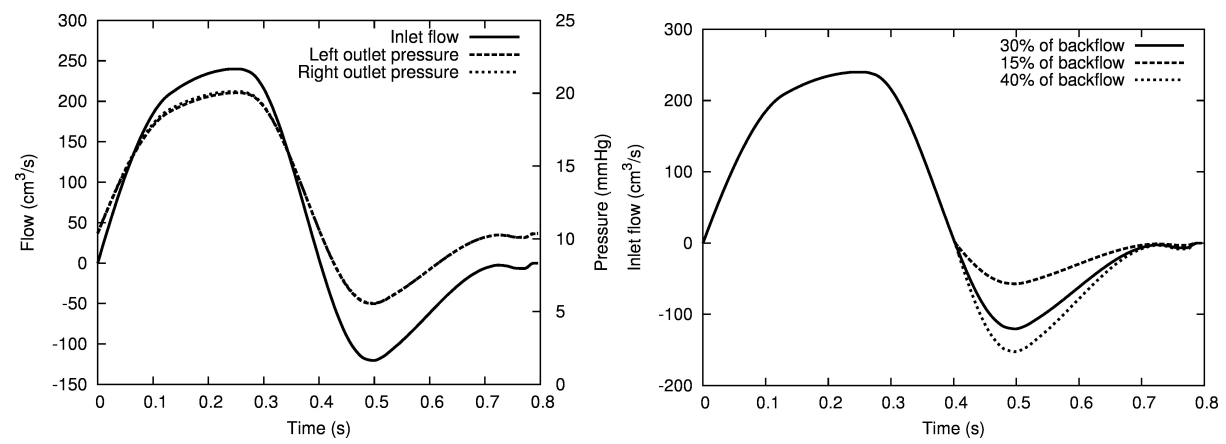

Figure 9: Left. Inlet flow (mean inlet flow rate of $3.5 \mathrm{~L} \cdot \mathrm{min}^{-1}$ ) and outlet pressure for the atlas under pathological boundary conditions. Right pathological inlet flows with different backflows. The prescribed inlet flows with $15 \%$ and $40 \%$ of backflow respectively present a mean value of 4.3 and $3.1 \mathrm{~L} \cdot \mathrm{min}^{-1}$.

are reported in Fig. 11. The error in velocity varies over time and among patients between $3 \%$ and $42 \%$, with an average curve rising from $7 \%$ to $27 \%$ and then decreasing slowly down back to $7 \%$ (mean over time being 15\%). Pressure errors vary over time and among patients from negligible values to $14 \%$, typically rising quickly to its peak value and decreasing equally fast to very low values and remaining low for the rest of the cycle. The mean over time is thus $1 \%$. Pressure loss errors follow similar pattern as the pressure, with a peak over time and among patients at $45 \%$, and an average among patients and over time of $5 \%$.

Fig. 12 shows the errors (17)-(18) for the different patients under pathological flow conditions. The corresponding mean errors and standard deviations are further reported in Fig. 13. We obtained 


\begin{tabular}{|c|c|}
\hline$R_{p}\left(\right.$ dyn $\left.\cdot \mathrm{s} \cdot \mathrm{cm}^{-5}\right)$ & 40 \\
\hline $\mathrm{C}\left(\mathrm{cm}^{5} \cdot \mathrm{dyn}^{-1}\right)$ & $10^{-3}$ \\
\hline $\mathrm{R}_{\mathrm{d}}\left(\mathrm{dyn} \cdot \mathrm{s} \cdot \mathrm{cm}^{-5}\right)$ & 300 \\
\hline
\end{tabular}

\begin{tabular}{|c|c|}
\hline$R_{p}\left(\mathrm{dyn} \cdot \mathrm{s} \cdot \mathrm{cm}^{-5}\right)$ & 107 \\
\hline $\mathrm{C}\left(\mathrm{cm}^{5} \cdot \mathrm{dyn}^{-1}\right)$ & 0.32 \\
\hline $\mathrm{R}_{\mathrm{d}}\left(\mathrm{dyn} \cdot \mathrm{s} \cdot \mathrm{cm}^{-5}\right)$ & 308 \\
\hline
\end{tabular}

Table 1: RCR Windkessel model values imposed at each outlet for normal (left) and pathological inlet flow conditions (right).
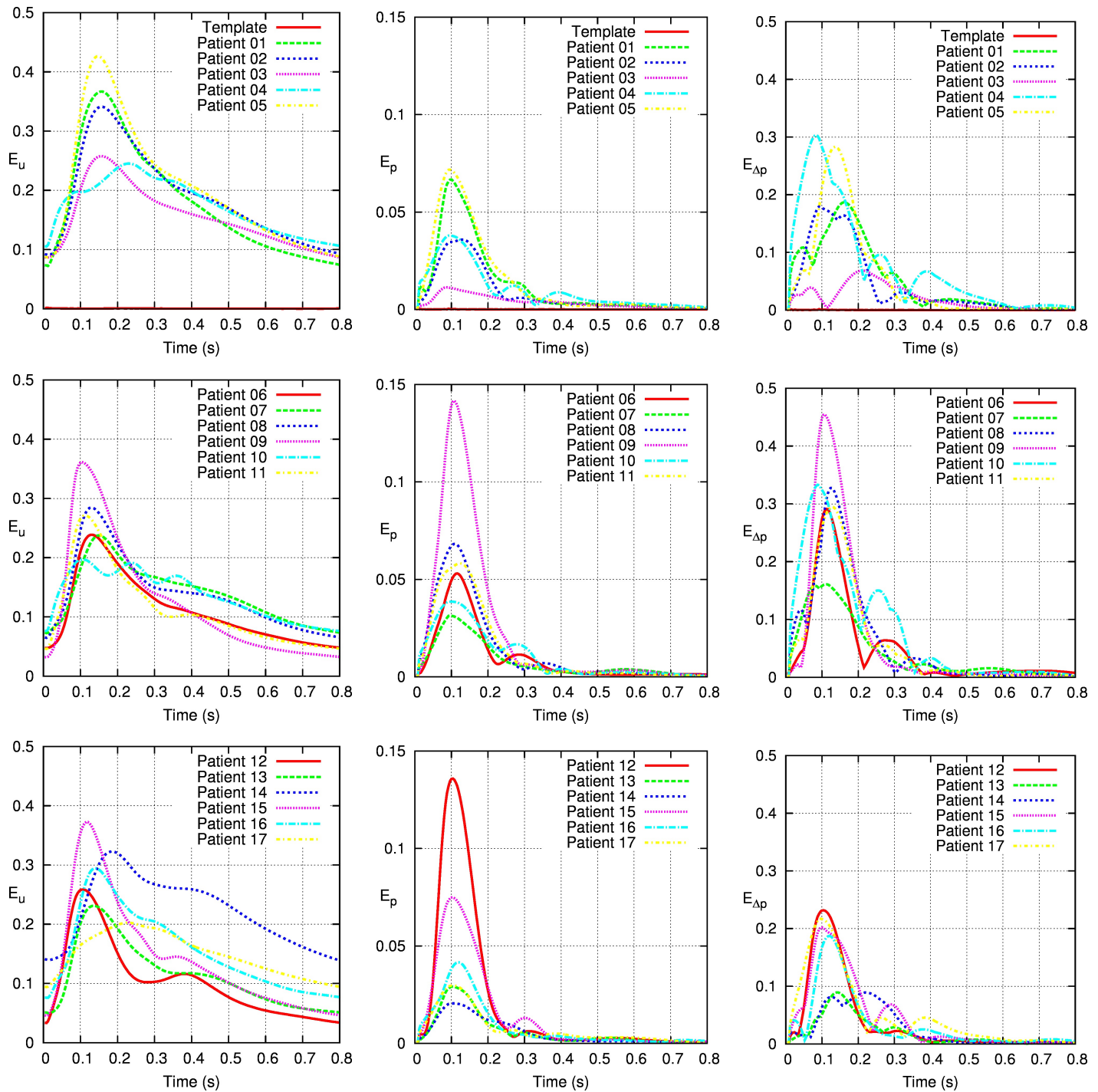

Figure 10: Velocity (left), pressure (center) and pressure drop (right) errors (computed according to equations (17)-(18)), between the full and the reduced order solutions for the same normal boundary conditions. 

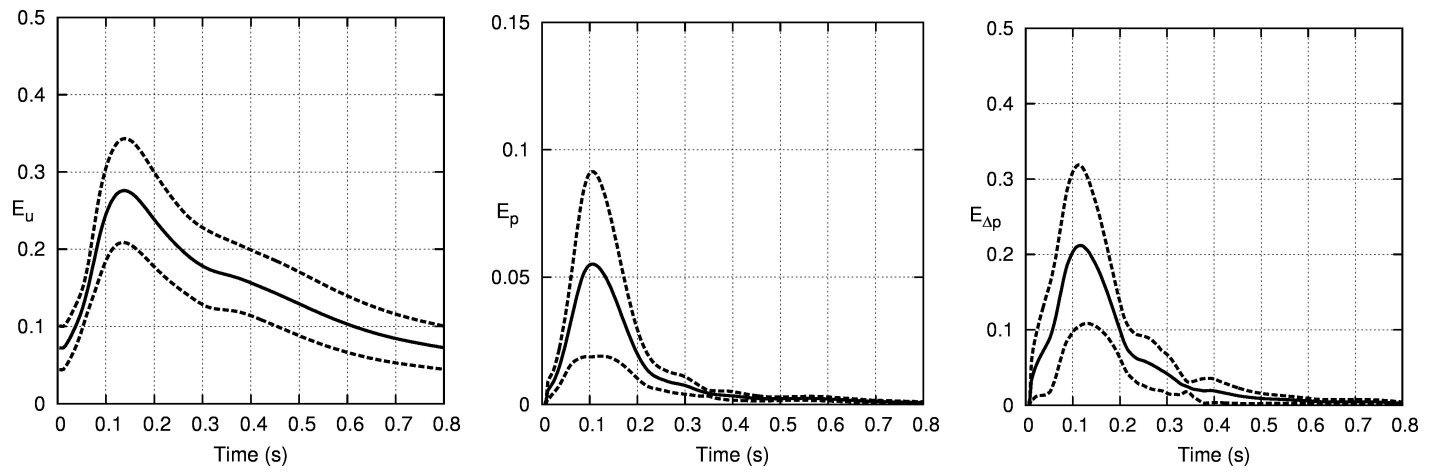

Figure 11: Mean (solid line) and standard deviation (dashed line) for velocity (left), pressure (center) and pressure drop (right) errors (computed according to equations (17)-(18)) between the full and the reduced order solutions with the same normal boundary conditions.

an error in velocity that varies over time and among patients between $10 \%$ and $47 \%$, with an average curve going from $18 \%$ to $30 \%$ with a double-bump waveform (mean in time being $23 \%$ ). Conversely, the errors in pressure vary over time and among patients from negligible values to below $5 \%$, also with a double-bump waveform but with a much lower second peak. The mean over time is $1 \%$. Pressure loss errors follow a four-bump pattern, with a negligible minimum value and a peak of $40 \%$ over time and amongst patients, and an average over patients and time of $11 \%$.

Figure 14 shows mean errors and standard deviations for the outlet flows (equation (19)) for normal and pathological inlet boundary conditions. In both cases, the mean error over all patients and over time is close to $2.5 \%$. Notice that as the imposed inlet flows are identical for full and reduced simulations, evaluating the amount of outlet flows allows to measure indirectly the accuracy of the reduced calculations regarding the flow split between the two branches.

To better visualize the underlying differences of the 3D fields, velocity magnitude 3D-cuts of the full and the reduced simulations are presented for two representative patients under normal and pathological conditions respectively, at different times in the cardiac cycle. In Fig. 15, one can see that the main features of velocity magnitude are well captured in the reduced simulation with respect to the full simulation for patient 7 under normal conditions. There are larger differences during deceleration (2nd row), in coherence with the profile of the $L^{2}$-error (Fig. 10). Fig. 16 shows that the main features of velocity magnitude are also well captured in the reduced simulation with respect to the full simulation for patient 13 under pathological conditions. In accordance with the 

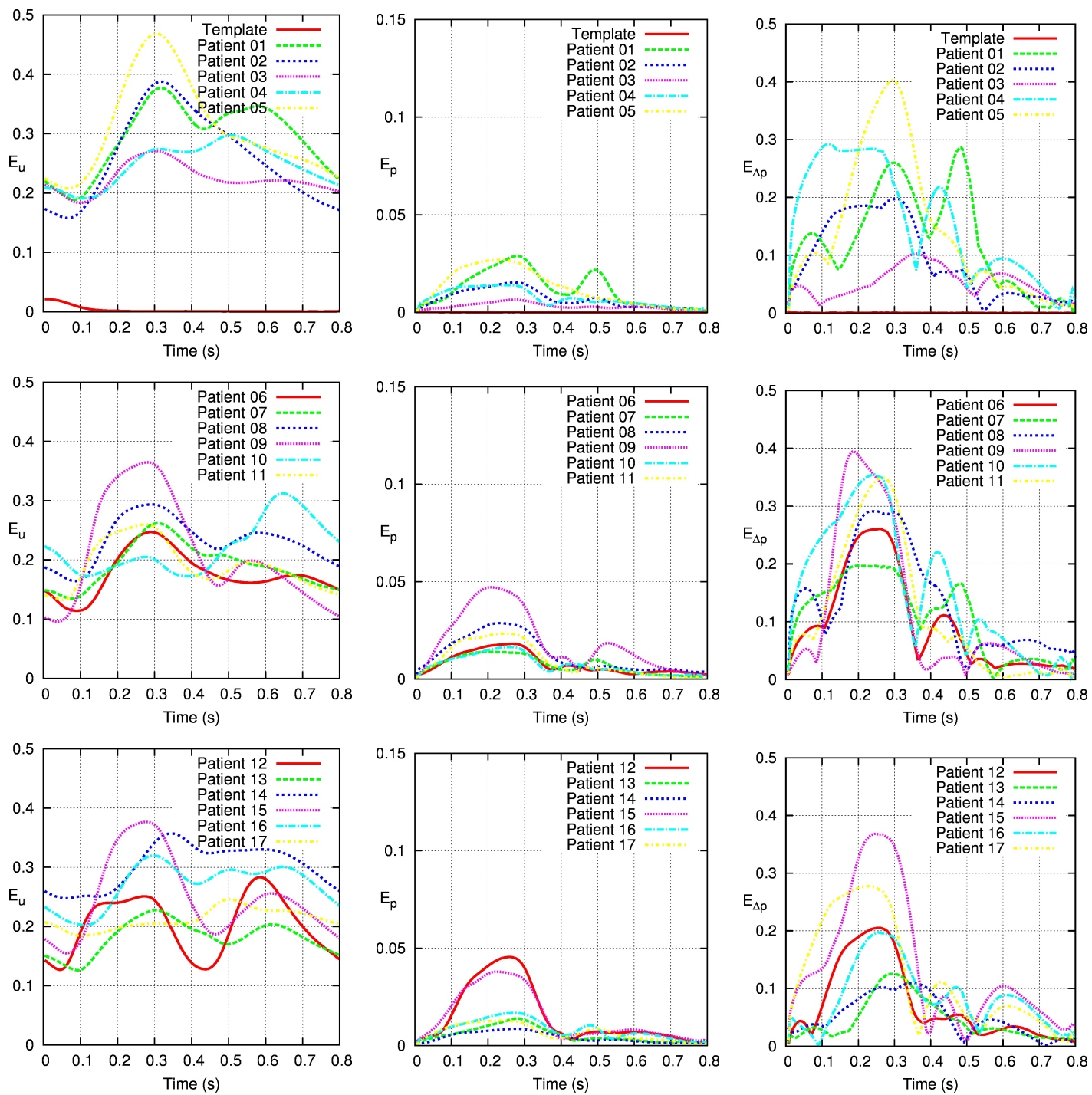

Figure 12: Velocity (left), pressure (center) and pressure drop (right) errors (computed according to equations (17)-(18)) between the full and the reduced order solutions for the same pathological boundary conditions. 

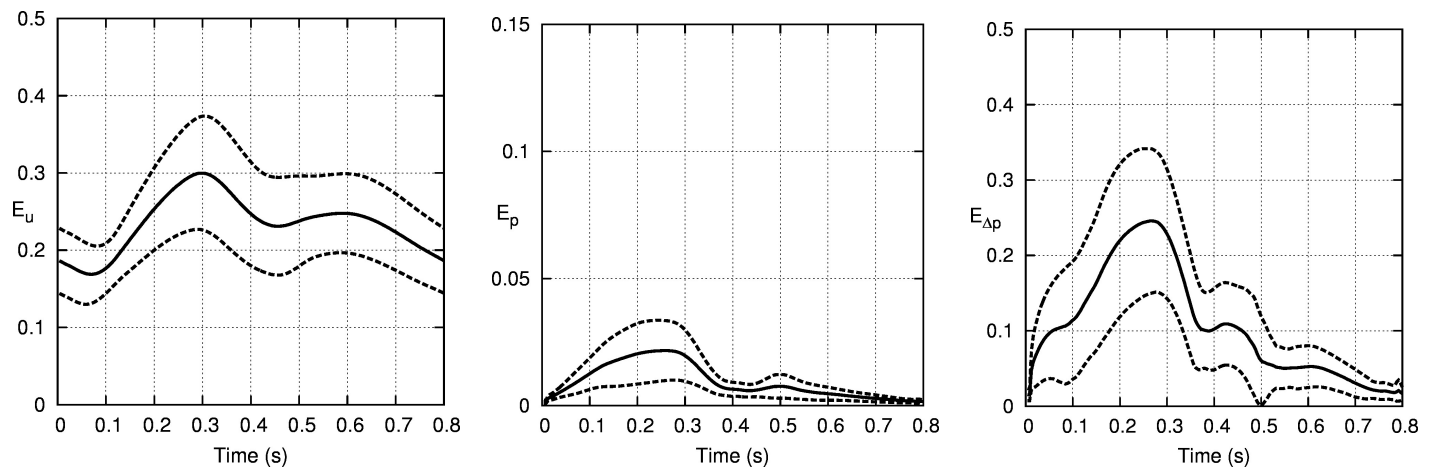

Figure 13: Mean (solid line) and standard deviation (dashed line) for velocity (left), pressure (center) and pressure drop (right) errors (computed according to equations (17)-(18)) between the full and the reduced order solutions for the same pathological boundary conditions.
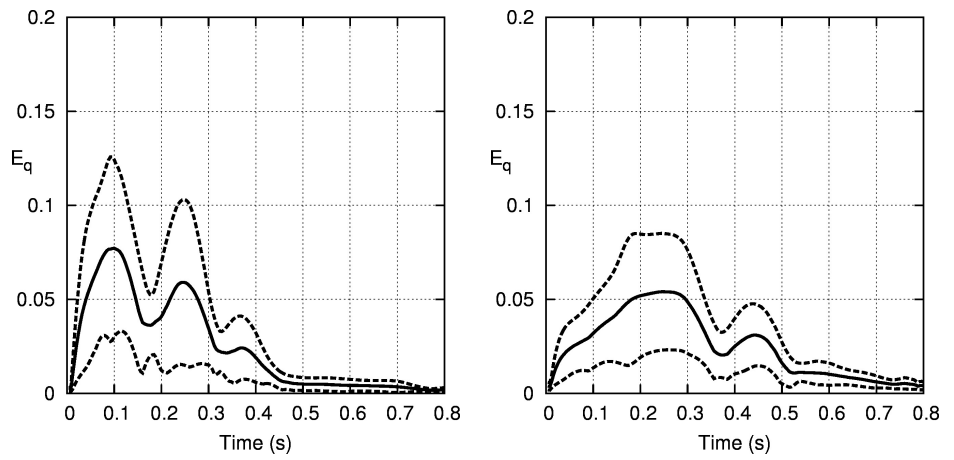

Figure 14: Mean (solid line) and standard deviation (dashed line) for outlet flow (computed according to equation (19)) between the full and the reduced order solutions under (left) normal and (right) pathological boundary conditions. 

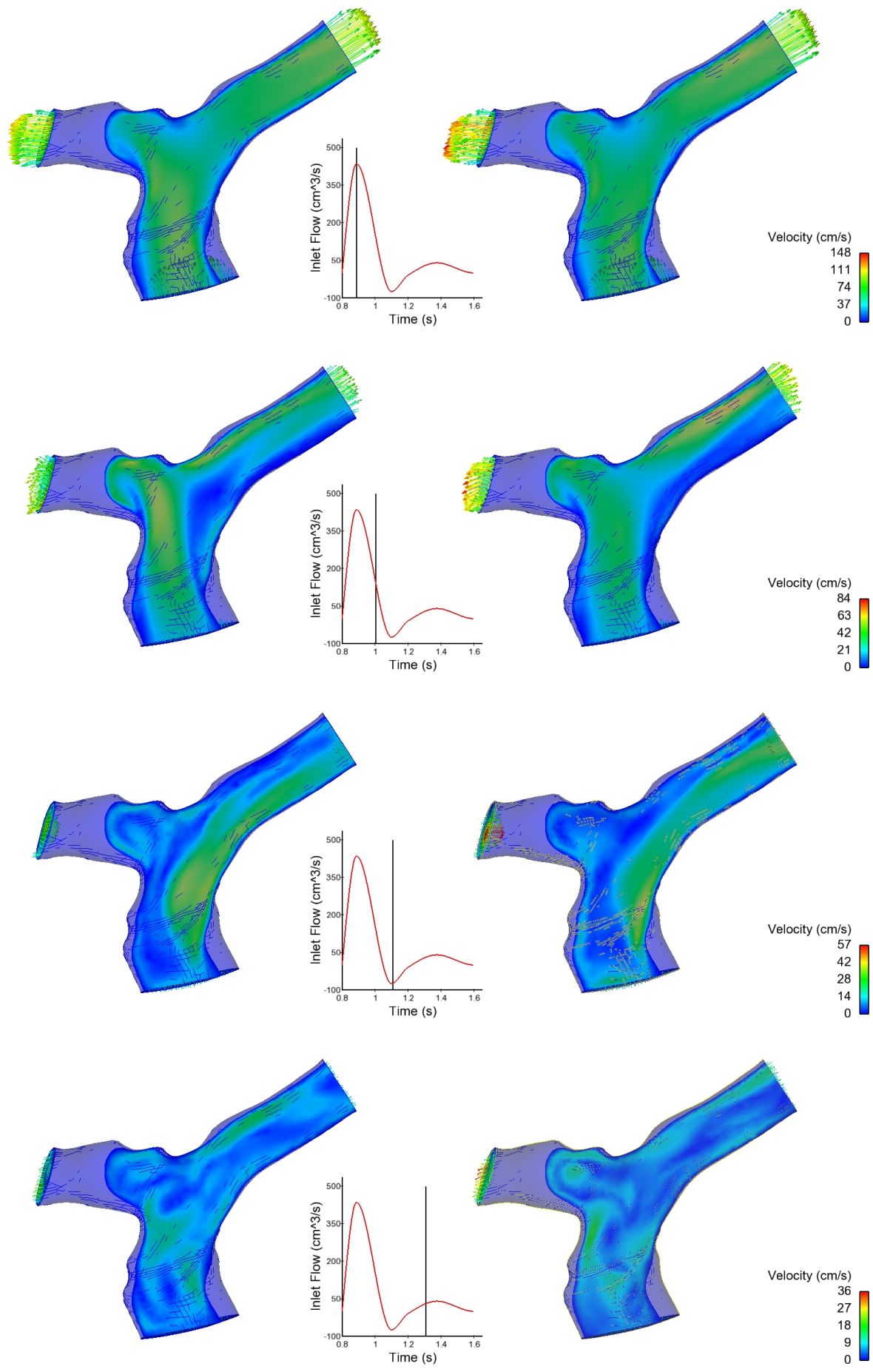

Figure 15: Full (left) and reduced (right) velocity fields for patient 07 under normal conditions shown at four instances along the cardiac cycle. 

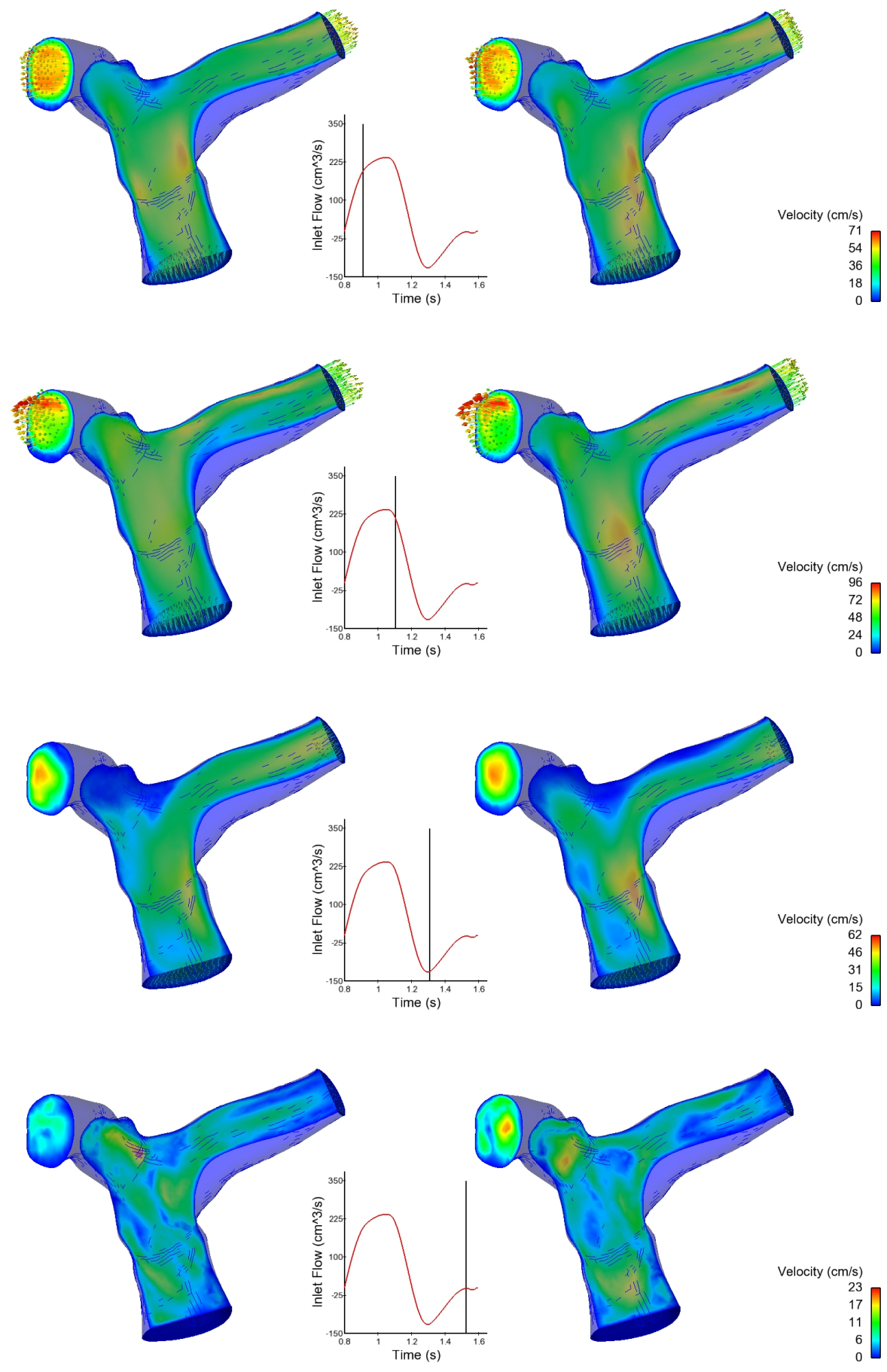

Figure 16: Full (left) and reduced (right) velocity fields for patient 13 under pathological conditions shown at four instances along the cardiac cycle. 
$L^{2}$-errors (Fig. 12), that do not vary much in time but peak at the beginning of deceleration, we observe the highest difference during the beginning of the deceleration (2nd row), although the four snapshots all show differences. For both normal and pathological flows, flow jets through the main pulmonary artery during peak systole, and presents complex structures in the pulmonary arteries at peak backflow and subsequent diastole.

\subsubsection{Simulations during exercise based on the resting conditions POD}

The inlet boundary conditions for the reduced simulations (step 5 of Algorithm 3) were modified to simulate normal and pathological conditions under exercise regimes, increasing heart rate and systolic flows (Fig. 17). The resulting average flow rates are $7.5 \mathrm{~L} \cdot \mathrm{min}^{-1}$ in the normal case and

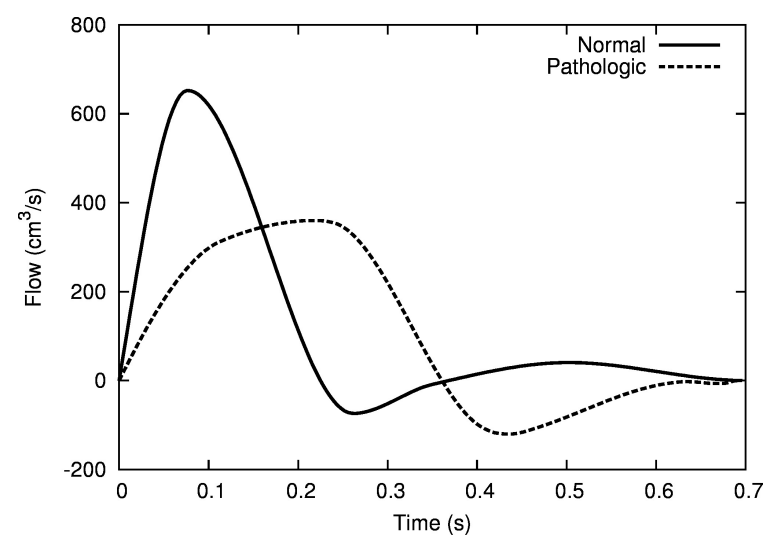

Figure 17: Normal and pathological inlet flows under exercise. Average flow rates are $7.5 \mathrm{~L}^{-}$min ${ }^{-1}$ in the normal case and $6.2 \mathrm{~L} \cdot \min ^{-1}$ with $20 \%$ backflow in the pathological case.

$6.2 \mathrm{~L} \cdot \mathrm{min}^{-1}$ with $20 \%$ backflow in the pathological case. For the reduced simulations, we used the reference POD basis computed in normal conditions, thus without performing an additional full computation on the reference geometry with the new boundary conditions. Fig. 18 summarizes the results for the different patients. The error in velocity on average rises from $7 \%$ to $30 \%$ and then decreases slowly back to $7 \%$. Pressure errors vary over time on average from negligible values to $7.5 \%$, typically rising rapidly to its peak value and decreasing equally quickly to very low values and remaining low for around $60 \%$ of the cycle. Pressure loss errors follow similar pattern as the pressure, with a peak at $25 \%$. Overall, the mean and standard deviation time variations and average are very similar to the resting conditions. Figure 19 displays the results of the exercise reduced simulations for pathological conditions. As in the normal case, we used the POD basis computed on 

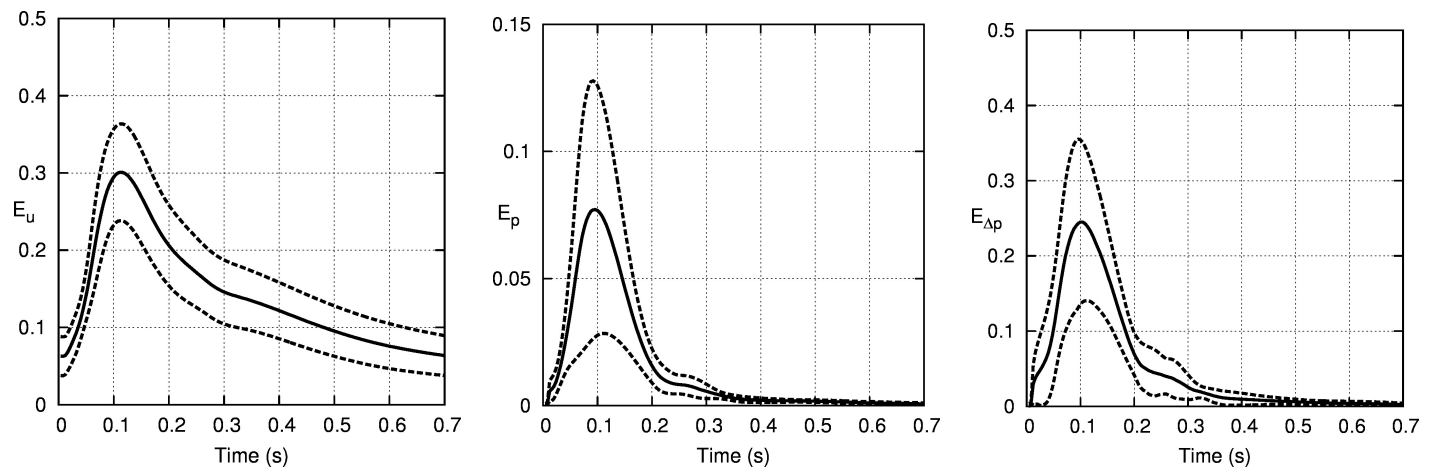

Figure 18: Mean (solid line) and standard deviation (dashed line) for velocity (left), pressure (center) and pressure drop (right) errors (computed according to equations (17)-(18)) between the full and the reduced order solutions under normal exercise boundary conditions (computed with the resting condition POD basis).

the atlas under rest conditions (see Remark 4.1). In this case, the error in velocity on average rises from $14 \%$ to $32 \%$, then decreasing with a small second bump back to $14 \%$. Pressure errors vary over time from negligible values to $3 \%$, remaining very low for around $60 \%$ of the cycle. Pressure loss errors follow a four-bump evolution, with a peak at $28 \%$. Overall the mean and standard deviation time variations are similar to resting conditions, but with less pronounced secondary bumps.
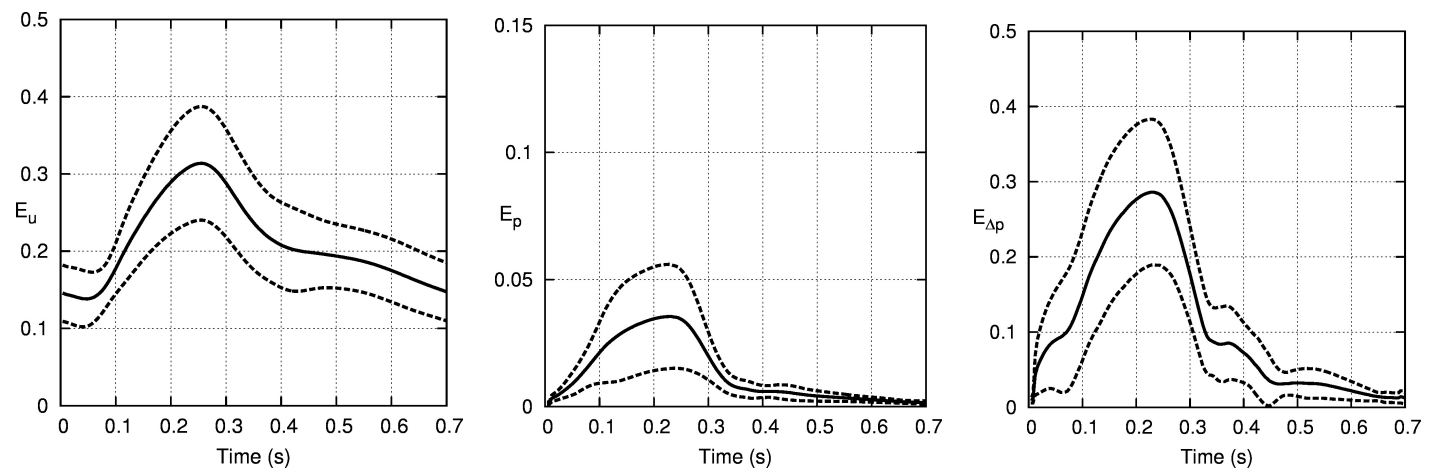

Figure 19: Mean (solid line) and standard deviation (dashed line) for velocity (left), pressure (center) and pressure drop (right) errors between the full and the reduced order solutions under pathological exercise boundary conditions (computed with the pathological resting conditions POD basis).

\subsubsection{Simulations of different pathological conditions based on the reference pathological POD}

To further test the robustness of the POD approach, the atlas POD basis computed with $30 \%$ backflow was used as reduced basis for the flow in the different patients under other pathological 
conditions, considering $15 \%$ and $40 \%$ of backflow. Mean errors and standard deviation results for these pathological conditions are respectively shown in Fig. 20 and Fig. 21. During systole, the flow
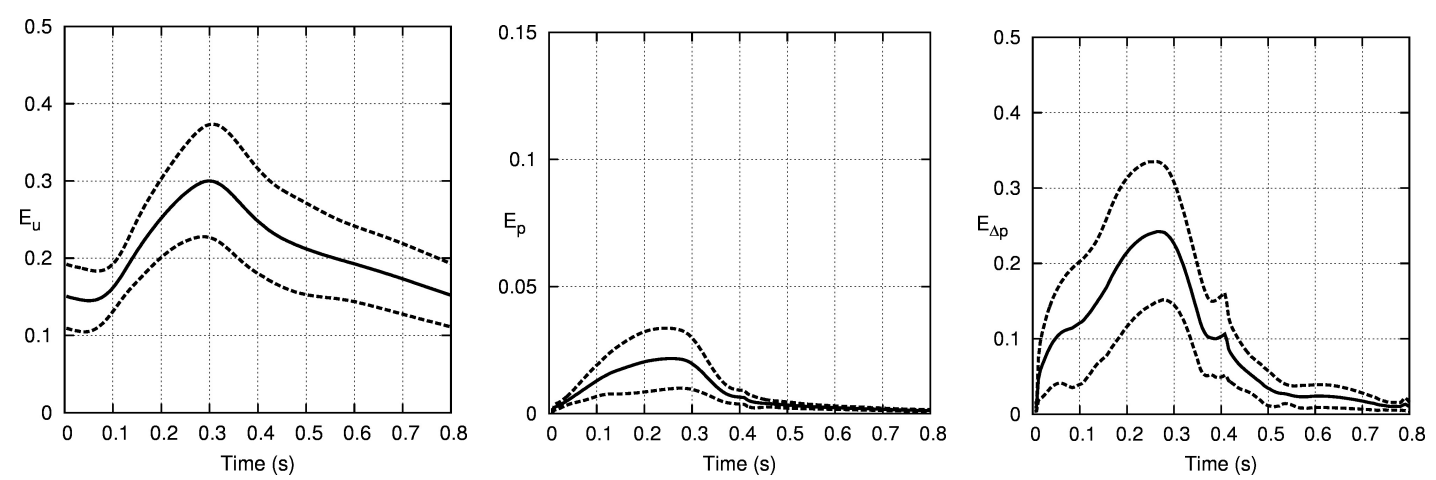

Figure 20: Mean (solid line) and standard deviation (dashed line) for velocity (left), pressure (center) and pressure drop (right) errors between the full and the reduced order solutions using pathological boundary conditions with $15 \%$ of backflow (using original pathological POD bases obtained with $30 \%$ of backflow).

conditions are the same for all the pathological conditions, and there is no increase of error during that period. In fact, pressure losses even decrease for the $40 \%$ backflow case. During diastole, the $15 \%$ flow reversal case has a decrease in all errors compared to the $30 \%$ flow reversal case on which the POD was constructed. The velocity error decays at the same rate as for normal flow conditions. The averages over patients and time are $21 \%$ for velocity, $1 \%$ for pressure and $9 \%$ for pressure loss. For the higher flow reversal case $(40 \%)$, there is an increase in errors (especially in velocity) after peak flow reversal. The velocity error has a second bump as high as the first one, even though in absolute value peak backflow is not as high as peak forward flow, and similarly for its derivative (re-acceleration versus deceleration). Pressure errors are, on the other hand, only slightly higher. The averages over patients and time are $26 \%$ for velocity, $1 \%$ for pressure and $10 \%$ for pressure loss.

\subsubsection{Simulations of the flow on the original patient meshes}

Since there are no experimental data to compare the reduced model results with, the simulations on the original patient meshes can be considered as the "ground truth". It is then natural to wonder what additional error is introduced by this small geometrical change between the original patient mesh and the atlas-to-patient deformed mesh (the registered patient mesh), and how it compares to the overall error, i.e. the difference between the reduced order solution computed in the sections 

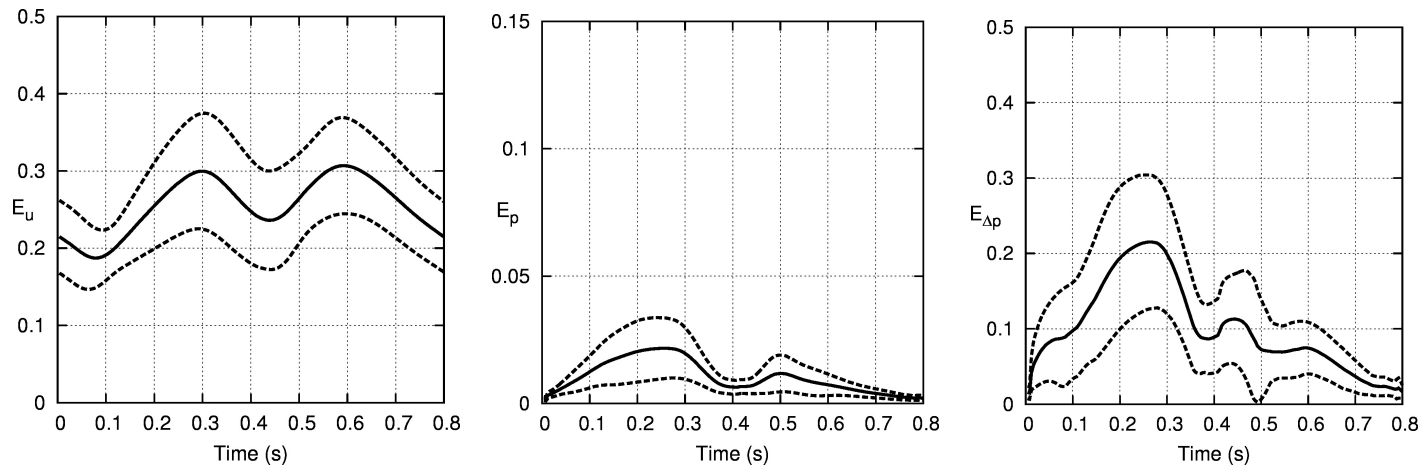

Figure 21: Mean (solid line) and standard deviation (dashed line) for velocity (left), pressure (center) and pressure drop (right) errors between the full and the reduced order solutions using pathological boundary conditions with $40 \%$ of backflow (using original pathological POD bases obtained with $30 \%$ of backflow).

above, and the solution on the original patient mesh. Studying these errors in detail would require to interpolate between 3D meshes of different topologies, resulting in a much harder procedure in practice, that can introduce an artificial additional error. Thus, for a restricted set of patients under normal conditions, additional simulations were run on the original patient meshes. Integrated quantities such as the pressure drop and the outflow distribution errors were computed; for such quantities a point-to-point mesh correspondence is not needed. These preliminary results (Table 2) show that the error due to the change of geometry is of the same order of magnitude as the overall error and the reduced order modeling error extensively studied in the sections above.

\begin{tabular}{|r|c|c|}
\hline Pressure drop & Error original vs. full & Error original vs. reduced \\
\hline \hline Patient 7 & 0.055 & 0.043 \\
\hline Patient 13 & 0.051 & 0.061 \\
\hline \hline Outlet flow & Error original vs. full & Error original vs. reduced \\
\hline \hline Patient 7 & 0.032 & 0.040 \\
\hline Patient 13 & 0.020 & 0.023 \\
\hline
\end{tabular}

Table 2: Average errors (instantaneous errors computed according to equations (18)-(19), averaged over one cardiac cycle) for the pressure drop and the outflow errors of two patients under normal conditions. Original corresponds to the full solution on the original patient mesh, full to the solution on the registered patient mesh, and reduced to the reduced order solution on the registered patient mesh. 


\section{Discussion}

\subsection{Results of reduced flow simulations}

The reduced model framework has been tested with 17 tetralogy of Fallot patients to create the atlas as a centered reference geometry. Although the pipeline has been designed to simulate reduced-order blood flow on new patients, in this work we tested the method on the same patients the atlas was created from. This is justified by the fact that the atlas was shown to be unbiased (see the leave-one-out validation step of Fig. 6), and since the POD basis is computed solely on the atlas geometry, this should have a minor effect on the conclusions of the study. From a qualitative point of view, the numerical tests show that the flow dynamics can be well captured by the reduced model in both normal and pathological conditions ${ }^{2}$. However, when looking at velocity magnitude maps at specific times and with their own scales, Fig. 15 and 16, differences can be more easily seen.

\subsubsection{Normal flow conditions}

Let us consider the case of blood flow simulations under normal conditions (Fig. 11). In this situation, the error is lowest for pressure with maximum in general below 10\% (Fig. 10), and an average over patients and time of $1 \%$. Higher errors were in general obtained for pressure drop. The peaks of these errors are located at the beginning of flow deceleration, where flow patterns appear to be more dependent on the geometry. However, the average over time of the mean error in pressure drop (Fig. 11) is 5\% only. Concerning the velocity field, the error norm in time resulted between $3 \%$ and $42 \%$, with relatively high variability among subjects (Fig. 10). Despite the high peaks, velocity errors stay limited for most of the cycle, and the average over time of the mean curve (Fig. 11) is only 15\%. In general, note that the error is not proportional to flow, and, as for the pressure and pressure drop, the maximum error occurs during early deceleration. Moreover, the error does not decrease to zero when flow does, but it rather decreases when the flow approaches a steady state, and velocity is lower in the whole domain.

\footnotetext{
${ }^{2}$ See supplementary movie material Patient_3_NormalConditions.avi, Patient_3_PathologicalConditions.avi.
} 


\subsubsection{Pathological flow conditions}

Next, we studied the performance of the reduced order model for pathological boundary conditions, employing for individual simulations a mapped POD basis computed with the same conditions on the reference geometry. In this case, the peak pressure error is twice lower than in the normal case, with a lower variability between the subjects (lower standard deviation in Fig. 13). But the time average remains at $1 \%$. Unlike the case of normal conditions, in this situation the average error follows closely the absolute flow time variation, with the highest bump occurring during high flow (or high pressure, which in this case is almost in phase with flow, see Fig. 9). At the same time, the error decrease is less evident than in the normal case, and the error increases again in correspondence to peak flow reversal, which is higher in this pathological condition. In contrast with the pressure field, the velocity and pressure loss errors under pathological flow conditions are larger (increase on average from $15 \%$ to $23 \%$, and from $5 \%$ to $10 \%$ respectively), with more variability among subjects on the magnitude and timing of the error extrema (Fig. 10). This suggests that the multiple changes of direction in the flow accentuate geometrical differences and lead to more complex velocity patterns, more sensitive to the individual geometries.

\subsubsection{Exercise boundary conditions}

Finally, reduced simulation under exercise conditions were performed with the POD generated under rest conditions. The velocity errors are very comparable on average, with similar time variation and standard deviation as under rest, for both the normal and pathological conditions. This is quite remarkable, since flow peak increases by $50 \%$ in the normal case and even by $100 \%$ in the pathological case. Concerning pressure, peak errors in normal conditions generally increase, but remain on the order of $10 \%$, while errors in pressure drop increase slightly. These trends are more pronounced for the pathological regime, with less effect of flow reversal compared to the rest condition, coherent with the fact that there is less backflow under the exercise condition. On average over time however, pressure and pressure drop errors do not change and thus remain low for both conditions.

\subsection{Limitations and perspectives}

The present study shows that the atlas-based reduced order model is able to capture the main hemodynamic features of the flow for a reasonable range of boundary conditions (e.g., rest versus exercise conditions, different degrees of pathologies). 
In particular, results point out the capability of the reduced order method to approximate the pressure fields in different geometries (the time average errors are $1 \%$ for pressure and $5-10 \%$ for pressure losses). In fact, since pressure represents the main component of the fluid force, this feature could be applied to perform efficient numerical studies of medical device design and stability under varying flow conditions, considering different configurations as deformations of a reference domain.

\subsubsection{POD for different flow regimes}

Nevertheless, the study of different inlet boundary conditions underlines the sensitivity of the errors to the flow regime (average errors, timings of peak errors), suggesting the importance of using a reference POD basis computed under the same regime (normal or pathological conditions respectively). Within a regime however, the POD generated for rest boundary conditions yielded satisfactory results also in the case of exercise conditions. Similarly, a POD generated in a pathological case successfully simulated other degrees of pathology . The importance of the POD basis flow regime has been confirmed by further numerical studies (results not shown), which demonstrated that the POD basis computed with normal boundary conditions does not approximate well the fluid dynamics under pathological conditions. Moreover, we observed that a POD basis combining snapshots of both normal and pathological simulations did not yield any gain (nor loss) in the accuracy (results not shown) of the simulation in the pathological regime.

\subsubsection{Relevance of geometry variability}

Another important observation concerns the increase of the error among patients for velocity compared to pressure, especially under complex flow conditions. This for now precludes the use of the method if detailed hemodynamics quantities are sought, such as wall shear stress. In addition, since complex flow patterns are more sensitive to the domain shape, this suggests that the main contribution to the error comes from the mismatch between the atlas and individual geometries. The influence of the variability of the geometry on the approximation properties of the POD basis remains an open issue. In particular, identifying the geometric parameters at the core of the highest errors would allow a great advance in the understanding of the flow characteristics and in the construction of better POD bases. However, identifying those that robustly describe flow disturbances is not an easy task (Bijari et al., 2012). 
A key point is that the reduced order method has been tested with blood flow solutions, for different regimes, always computed on a single geometry. This surely limits the information contained in the reference POD basis, reducing the ability of the transported POD to capture the full flow dynamics on different geometries. In order to decrease the error, a possible extension of the method could consider the use of multiple atlas geometries (from different populations) and the computation of different POD bases, which could be combined when building the reduced models for the flow simulations on new patients. Ultimately, we would like to better understand the impact of the geometric features on the flow. However, to this aim, a larger number of patients is needed in order to obtain statistically significant results.

\subsubsection{Reduced vs. full simulations}

As noted earlier, the full CFD simulations on the individual geometries, used for benchmarking the POD reduced models, were based on the geometries obtained by registering the atlas to each patient (3D-shape registration, described in Section 3.2.1), i.e. the same geometries used for the reduced simulations. This results in a discrepancy between the original meshes and the atlas-topatient deformed meshes, since we allowed for some noise in the atlas construction step. However, due to the fact that the atlas is well-centered, these differences are geometrically small. We kept separated the error due to the noise in the atlas computation procedure from the error introduced by the transported model reduction. Addressing the first error in detail would require an additional interpolation between meshes with different topologies. For a restricted set of patients, we have monitored the pressure drop and flow split errors with respect to the original geometries. These quantities can be easily compared, even on different meshes. The results have shown that the errors due to the noise in the atlas construction step are of the same order of magnitude as the errors due to the transported model reduction. Future work should confirm these results on a larger set of patients and conditions.

In fact, this question raises the more general question of validation. It would be interesting to compare the error between the reduced and full simulations, and the error between in vivo data and these CFD simulations (Arzani et al., 2012). However, with the current measurement technologies, this remains a very challenging task (this would necessitate e.g. real-time MRI velocity information or flow rate in different locations, pressure measurements recorded at the same time with precision of $1 \mathrm{mmHg}$ or less). Such comparison might be more easily done with in-vitro data (Kung et al., 
2011; Pekkan et al., 2009).

As a final remark, we observe that the main focus of the paper was to assess the proposed atlasbased reduced order modeling in terms of capability to reproduce different scenarios (e.g., from different geometries, boundary conditions), without directly discussing the issue of computational time. It is important to remind that, even when the number of degrees of freedom is drastically reduced (from about $10^{5}$ to a few dozen in our case), the nonlinearities may compromise the reduction of computational time if they are not correctly handled. A number of works have addressed this issue, see, e.g., (Carlberg et al., 2011; Grepl et al., 2007; Baiges et al., 2012; Wang et al., 2012; Yano et al., 2012; Grinberg et al., 2009; Grinberg, 2012). With the current implementation of our method, we observed a reduction of $20 \%$ to $30 \%$ of the CPU time with respect to the full order model. There is therefore still room for improvement, and this will be the topic of future work.

\section{Conclusion}

We presented a model order reduction approach, which combines statistical shape analysis for the computation of a reference geometry and proper orthogonal decomposition (POD). The reduced model framework has been tested with 17 tetralogy of Fallot patients to create the atlas as a centered reference geometry. Reduced simulations were computed on these same patients. In order to apply the procedure for the reduced simulation on new patients, the framework requires simply the surface mapping between the atlas and the new geometry, in order to compute the specific reduced basis.

Numerical experiments show that the algorithm yields errors that are acceptable for applications that need to capture pressure and the main velocity features, as, e.g., in the study of medical devices. However, if velocity field or pressure loss are required with higher precision, more accurate approaches may be necessary, which take into account the geometric variability at a smaller scale. This is especially true under pathological conditions. Furthermore, our numerical simulations demonstrate that the reduced model is robust with respect to changes in boundary conditions, in particular to simulate exercise conditions from rest simulations, or different degrees of pathology.

\section{Appendix}

\subsection{Currents representation of surfaces}

The basic principle of the currents approach is to probe a surface by a set of vector fields in a space $W$, subset of the square integrable three-dimensional vector fields. In this way, a surface $S$ is 
represented by a current, which maps any vector field $\omega$ in $W$ to the flux of the vector through the surface:

$$
C_{S}(\omega)=\int_{S} \omega(x)^{t} \mathbf{n}(x) d \lambda(x) .
$$

In (20), $\mathbf{n}(x)$ is the unit normal of the surface at a point $x$, and $d \lambda$ is the Lebesgue measure on the surface. The space $W$, called the test space, is chosen as the set of convolutions between any square integrable vector field and a smoothing kernel. Hence, $W$ is a Reproducing Kernel Hilbert Space (RKHS). In this work we use a Gaussian kernel, which can be defined, for any points $(x, y)$, as

$$
K_{W}(x, y)=\exp \frac{\frac{-\|x-y\|^{2}}{\lambda_{W}^{2}}}{,}
$$

where $\lambda_{W}$ is the standard deviation. With this choice for the reproducing kernel, we can then control a metric in the space of currents that allows the distance between two surfaces to be efficiently computed, though other choices of kernels are possible. This space also has the important property that it is the dense span of basis vector fields of the form $\omega(x)=K_{W}(x, y) \beta$, for any fixed point $y$ and fixed vector $\beta$. As a consequence, any vector field $\omega$ can be written as an infinite linear combination of the basis elements $K_{W}(x, y) \beta$. We can also define an inner product in $W$ using the kernel $K_{W}$ with these basis vectors as

$$
\left\langle K_{W}(., x) \alpha, K_{W}(., y) \beta\right\rangle_{W}=\alpha^{t} K_{W}(x, y) \beta .
$$

This inner product holds for any vector field $\omega(x)=K_{W}(x, y) \beta$ in $W$.

The space of currents is defined as the dual space of $W$, denoted $W^{*}$, representing the vector space of linear mappings from $W$ to $\mathbb{R}$. Since $W$ is a Reproducing Kernel Hilbert Space, the evaluation functionals are bounded. From the properties of $W$ (the fact that $W$ is densely spanned by the vector fields $\omega(x)$, and has an associated inner product) its dual space $W^{*}$ is densely spanned by the dual representations of the basis vectors $\omega(x)$, called the Dirac delta currents, defined as:

$$
\left\langle\delta_{x}^{\alpha}, \omega\right\rangle_{W}=\left\langle K_{W}(x, .) \alpha, \omega\right\rangle_{W}=\alpha^{t} \omega(x)
$$

Given that $W^{*}$ is a vector space, we can define the sum of two surfaces (represented by currents) $C_{S_{1}}$ and $C_{S_{2}}$ (obtained from equation (20)) as $\left(C_{S_{1}}+C_{S_{2}}\right)(\omega)=C_{S_{1}}(\omega)+C_{S_{2}}(\omega)$. In terms of the flux, this means that the flux through the sum of two surfaces is the sum of the flux through each surface. The vector space property of scalar multiplication in this case means that we can scale a 
current by simply scaling the Dirac delta currents. We can also define an inner product in $W^{*}$ as:

$$
\left\langle\delta_{x}^{\alpha}(\omega), \delta_{y}^{\beta}(\omega)\right\rangle_{W^{*}}=\left\langle K_{W}(x, .) \alpha, K_{W}(y, .) \beta\right\rangle_{W}=\alpha^{t} K_{W}(x, y) \beta
$$

A key advantage of the currents representation is that a metric can be defined which does not assume point correspondences between surfaces. Rather than measuring the distances between points on the surface, surfaces are compared at an anatomical level. The distance between two surfaces can then be expressed as the norm of the difference between the surfaces, which is the distance between their currents:

$$
\left\|C_{S_{1}}-C_{S_{2}}\right\|_{W^{*}}
$$

\section{2. $L D D M M$ framework for inter-patient surface registration}

The LDDMM framework uses a group of diffeomorphisms constructed through the integration of time-varying velocity fields, determined by the initial velocity at time $t=0$ parameterized by moment vectors, that belong to a RKHS. This yields a diffeomorphism $\phi$ as well as a differentiable flow of the diffeomorphism $\phi_{k}$ for a continuous parameter $k$ within the interval $[0, t]$. At time $k=0$, we have the identity mapping $\phi_{0}$. The mapping at time $t$ gives the desired transformation $\phi_{t}$ which we require for the atlas estimation. We can then define the path at any point $x$ as $\phi_{k}(x)$ that leads to the final position $\phi_{t}(x)$. By following the path that passes through the point $x$, we can compute easily operations on diffeomorphisms, such as the inverse path from point $t$ to 0 . Using this framework, we can minimize the difference between a deformed surface $\phi_{t}\left(S_{1}\right)$ and another surface $S_{2}$, therefore finding the geodesic path from $\phi_{t}\left(S_{1}\right)$ to $S_{2}$. The deformation $\phi_{t}$ is estimated by the minimisation of a weighted sum of two terms: $\varepsilon(\phi)=d^{2}\left(\phi_{t}\left(S_{1}, S_{2}\right)\right)+\lambda \int\left\|v_{t}\right\|^{2}$, the first

term is the distance between surfaces (measured in the space of currents), and the second is the energy of the trajectory in the space of deformations, with $\partial \phi_{t}(x) / \partial t=v_{t}(x), \phi_{0}(x)=x$. The $v_{t}(x)$ are time-varying velocity fields parameterised by the initial velocity field $v_{0}(x)$, which belongs to a RKHS generated by a Gaussian kernel $K_{V}(x, y)$. 


\section{REFERENCES}

Allassonnière, S., Amit, Y., Trouvé, A., 2006. Towards a coherent statistical framework for dense deformable template estimation. J.R. Statist. Soc.B 69, 2007.

Arzani, A., Dyverfeldt, P., Ebbers, T., Shadden, S.C., 2012. In vivo validation of numerical prediction for turbulence intensity in an aortic coarctation. Ann Biomed Eng 40, 860-870.

Baiges, J., Codina, R., Idelsohn, S., 2012. Explicit reduced order models for the stabilized finite element approximation of the incompressible Navier-Stokes equations. Submitted preprint.

Bazilevs, Y., Hsu, M.C., Benson, D.J., Sankaran, S., Marsden, A.L., 2009. Computational fluidstructure interaction: methods and application to a total cavopulmonary connection. Comp. Mech. 45, 77-89.

Beg, M., Helm, P., McVeigh, E., Miller, M., Winslow, R., 2004. Computational cardiac anatomy using MRI. Magn. Reson. Medicine 52, 1167-1174.

Bergmann, M., Bruneau, C., Iollo, A., 2009. Enablers for robust POD models. J. Comp. Phys. 228, $516-538$.

Bertoglio, C., Caiazzo, A., Fernández, M., 2013. Fractional-step schemes for the coupling of distributed and lumped models in hemodynamics. SIAM J. Sci. Comp. 35, B551-B575.

Bijari, P.B., Antiga, L., Gallo, D., Wasserman, B.A., Steinman, D.A., 2012. Improved prediction of disturbed flow via hemodynamically-inspired geometric variables. J. Biomech. 45, 1632-1637.

Biswas, R. (Ed.), 2010. Parallel Computational Fluid Dynamics: Recent Advances and Future Directions. DEStech Publications.

Blanco, P.J., Pivello, M.R., Urquiza, S.A., Feijoo, R.A., 2009. On the potentialities of 3d-1d coupled models in hemodynamics simulations. J. Biomech. 42, 919-930.

Cairncross, R., Schunk, P., Baer, T., Rao, R., Sackinger, P., 2000. A finite element method for free surface flows of incompressible fluids in three dimensions. Part I: Boundary fitted mesh motion. Int. Jour. Num. Meth. Fluids 33, 375-403. 
Carlberg, K., Bou-Mosleh, C., Farhat, C., 2011. Efficient non-linear model reduction via a leastsquares Petrov-Galerkin projection and compressive tensor approximations. International Journal for Numerical Methods in Engineering 86, 155-181.

Chai, L., Ding, J., Liu, Y., 2010. Hemodynamics Simulation of Patient-Specific Surgical Planning For Tetralogy of Fallot. 2010 3rd International Conference on Biomedical Engineering and Informatics, Yu, W. Zhang M. Wang L. Song Y.

Chern, M.J., Wu, M.T., Wang, H.L., 2008. Numerical investigation of regurgitation phenomena in pulmonary arteries of Tetralogy of Fallot patients after repair. J. Biomech. 41, 3002-3009.

Chorin, A., 1968. Numerical solution of the Navier-Stokes equations. Math. Comp. 22, 745-762.

Ciarlet, P., 1988. Mathematical elasticity. Vol. I. volume 20 of Studies in Mathematics and its Applications. North-Holland Publishing Co.

Das, A., Gottliebson, W.M., Karve, M., Banerjee, R., 2011. Comparison of hemodynamic endpoints between normal subject and tetralogy patient using Womersley velocity profile and MR based flow measurements. Mol. \& Cell. Biomech. 8, 21-42.

Durrleman, S., Pennec, X., Trouvé, A., Ayache, N., 2008. A Forward Model to Build Unbiased Atlases from Curves and Surfaces, in: Pennec, X., Joshi, S. (Eds.), Proc. of the International Workshop on the Mathematical Foundations of Computational Anatomy (MFCA-2008).

Durrleman, S., Pennec, X., Trouvé, A., Ayache, N., 2009. Statistical Models of Sets of Curves and Surfaces based on Currents. Med. Im. Anal. 13, 793-808.

Formaggia, L., Quarteroni, A., Vergara, C., . On the physical consistency between three-dimensional and one-dimensional models in haemodynamics. J. Comp. Phys. .

Frank, O., 1899. Die Grundform Des Arteriellen Pulses. Zeitung für Biologie 37, 483-586.

Grepl, M.A., Maday, Y., Nguyen, N.C., Patera, A.T., 2007. Efficient reduced-basis treatment of non affine and nonlinear partial differential equations. M2AN Math. Model. Numer. Anal. 41, 575-605.

Grinberg, L., 2012. Proper orthogonal decomposition of atomistic flow simulations. Journal of Computational Physics 231, 5542 - 5556. 
Grinberg, L., Cheever, E., Anor, T., Madsen, J.R., Karniadakis, G.E., 2011. Modeling blood flow circulation in intracranial arterial networks: A comparative 3d/1d simulation study. Ann Biomed Eng 39, 297-309.

Grinberg, L., Yakhot, A., Karniadakis, G.E., 2009. Analyzing transient turbulence in a stenosed carotid artery by proper orthogonal decomposition. Annals of biomedical engineering 37, 22002217.

Guermond, J.L., Minev, P., Shen, J., 2006. An Overview of Projection methods for incompressible flows. Comput. Methods Appl. Mech. Engrg. 195, 6011-6045.

Hsia, T.Y., Cosentino, D., Corsini, C., Pennati, G., Dubini, G., Migliavacca, F., Investigators, M.o.C.H.A.M., 2011. Use of mathematical modeling to compare and predict hemodynamic effects between hybrid and surgical norwood palliations for hypoplastic left heart syndrome. Circulation 124, S204-10.

Itu, L., Sharma, P., Ralovich, K., Mihalef, V., Ionasec, R., Everett, A., Ringel, R., Kamen, A., Comaniciu, D., 2013. Non-Invasive Hemodynamic Assessment of Aortic Coarctation: Validation with In Vivo Measurements. Ann. Biomed. Eng. 41, 669-681.

Keshavarz-Motamed, Z., Garcia, J., Maftoon, N., Bedard, E., Chetaille, P., Kadem, L., 2012. A new approach forthe evaluation of the severity of coarctation of the aorta using Doppler velocity index and effective orifice area: in vitro validation and clinical implications. J. Biomech. 45, $1239-1245$.

Kilner, P.J., Balossino, R., Dubini, G., Babu-Narayan, S.V., Taylor, A.M., Pennati, G., Migliavacca, F., 2009. Pulmonary regurgitation: The effects of varying pulmonary artery compliance, and of increased resistance proximal or distal to the compliance. Int. J. Cardiology 133, 157-166.

Koo, B.K., Erglis, A., Doh, J.H., Daniels, D.V., Jegere, S., Kim, H.S., Dunning, A., DeFrance, T., Lansky, A., Leipsic, J., Min, J.K., 2011. Diagnosis of ischemia-causing coronary stenoses by noninvasive fractional flow reserve computed from coronary computed tomographic angiograms. Results from the prospective multicenter DISCOVER-FLOW (Diagnosis of Ischemia-Causing Stenoses Obtained Via Noninvasive Fractional Flow Reserve) study. J. Am. Coll. Cardiol. 58, 1989-1997. 
Kung, E.O., Les, A.S., Figueroa, C.A., Medina, F., Arcaute, K., Wicker, R.B., McConnell, M.V., Taylor, C.A., 2011. In vitro validation of finite element analysis of blood flow in deformable models. Ann. Biomed. Eng. 39, 1947-1960.

LaDisa, J.F., Dholakia, R.J., Figueroa, C.A., Vignon-Clementel, I.E., Chan, F.P., Samyn, M.M., Cava, J.R., Taylor, C.A., Feinstein, J.A., 2011. Computational simulations demonstrate altered wall shear stress in aortic coarctation patients treated by resection with end-to-end anastomosis. Congenit. Heart Dis. 6, 432-43.

Løvgren, A., Maday, Y., Rønquist, E., 2007. The reduced basis element method for fluid flows, in: Analysis and simulation of fluid dynamics. Birkhäuser. Adv. Math. Fluid Mech., pp. 129-154.

Mansi, T., 2010. Image-Based Physiological and Statistical Models of the Heart, Application to Tetralogy of Fallot. Thèse de sciences (PhD Thesis). Ecole Nationale Supérieure des Mines de Paris.

Manzoni, A., Quarteroni, A., Rozza, G., 2011. Model reduction techniques for fast blood flow simulation in parametrized geometries. Int. J. Num. Meth. Biomed. Engnr. .

Markl, M., Geiger, J., Jung, B., Hirtler, D., Arnold, R., 2012. Noninvasive Evaluation of 3D Hemodynamics in a Complex Case of Single Ventricle Physiology. J. Magn. Reson. Imaging 35, 933-937.

McGregor, R., Szczerba, D., Muralidhar, K., Székely, G., 2009. A fast alternative to computational fluid dynamics for high quality imaging of blood flow. Medical Image Computing and ComputerAssisted Intervention-MICCAI 2009 , 124-131.

Mcgregor, R., Szczerba, D., von Siebenthal, M., Muralidhar, K., Székely, G., 2008. Exploring the use of proper orthogonal decomposition for enhancing blood flow images via computational fluid dynamics. Medical Image Computing and Computer-Assisted Intervention-MICCAI 2008 , $782-789$.

McLeod, K., Caiazzo, A., Fernández, M.A., Mansi, T., Vignon-Clementel, I.E., Sermesant, M., Pennec, X., Boudjemline, Y., Gerbeau, J.F., 2010. Atlas-Based Reduced Models of Blood Flows for Fast Patient-Specific Simulations, in: Proc. MICCAI Workshop on Statistical Atlases and 
Computational Models of the Heart: Mapping Structure and Function + a Cardiac Electrophysiological Simulation Challenge (STACOM+CESC'10), Springer, Beijing. pp. 95-104.

Moore, S.M., Moorhead, K.T., Chase, J.G., David, T., Fink, J., 2005. One-dimensional and threedimensional models of cerebrovascular flow. J. Biomech. Engnr.-Trans. Asme 127, 440-449.

Morales, H.G., Kim, M., Vivas, E.E., Villa-Uriol, M.C., Larrabide, I., Sola, T., Guimaraens, L., Frangi, A.F., 2011. How do coil configuration and packing density influence intra-aneurysmal hemodynamics? Am. J. Neuroradiol. 32, 1935-1941.

Pant, S., Limbert, G., Curzen, N.P., Bressloff, N.W., 2011. Multiobjective design optimisation of coronary stents. Biomaterials 32, 7755-7773.

Pekkan, K., Dasi, L.P., de Zélicourt, D., Sundareswaran, K.S., Fogel, M.A., Kanter, K.R., Yoganathan, A.P., 2009. Hemodynamic performance of stage-2 univentricular reconstruction: Glenn vs. hemi-Fontan templates. Ann Biomed Eng 37, 50-63.

Prasad, A., To, L.K., Gorrepati, M.L., Zarins, C.K., Figueroa, C.A., 2011. Computational analysis of stresses acting on intermodular junctions in thoracic aortic endografts. J. Endovasc. Ther. 18, $559-68$.

Puranik, R., Muthurangu, V., Celermajer, D.S., Taylor, A.M., 2010. Congenital Heart Disease and Multi-modality Imaging. Heart Lung and Circulation 19, 133-144.

Rathinam, M., Petzold, L., 2004. A new look at proper orthogonal decomposition. SIAM J Num. An. 41, 1893-1925.

Reymond, P., Perren, F., Lazeyras, F., Stergiopulos, N., 2012. Patient-specific mean pressure drop in the systemic arterial tree, a comparison between 1-d and 3-d models. J. Biomech. 45, 2499-2505.

Schwartz, M., Rome, J., Gillespie, M., Whitehead, K., Harris, M., Fogel, M., Glatz, A., 2011. Relation of Left Ventricular End Diastolic Pressure to Right Ventricular End Diastolic Volume After Operative Treatment of Tetralogy of Fallot. Am. J. Card. .

Spilker, R., Feinstein, J., Parker, D., Reddy, V., Taylor, C., 2007. Morphometry-Based Impedance Boundary Conditions for Patient-Specific Modeling of Blood Flow in Pulmonary Arteries. Ann. Biomed. Engnr. 35, 546. 
Temam, R., 1968. Une méthode d'approximation de la solution des équations de Navier-Stokes. Bull. Soc. Math. France 96, 115-152.

Tomasian, A., Krishnam, M.S., Lohan, D.G., Moghaddam, A.N., Finn, J.P., 2009. Adult Tetralogy of Fallot Quantitative Assessment of Pulmonary Perfusion With Time-Resolved Three Dimensional Magnetic Resonance Angiography. Investigative Radiology 44, 31-37.

Troianowski, G., Taylor, C.A., Feinstein, J.A., Vignon-Clementel, I.E., 2011. Three-Dimensional Simulations in Glenn Patients: Clinically Based Boundary Conditions, Hemodynamic Results and Sensitivity to Input Data. J. Biomech. Engnr.-Trans. Asme 133.

Vaillant, M., Glaunes, J., 2005. Surface matching via currents, in: Proc. IPMI 2005, Springer. p. 381.

Valverde, I., Parish, V., Tzifa, A., Head, C., Sarikouch, S., Greil, G., Schaeffter, T., Razavi, R., Beerbaum, P., 2011. Cardiovascular MR Dobutamine Stress in Adult Tetralogy of Fallot: Disparity Between CMR Volumetry and Flow for Cardiovascular Function. J. Magn. Res. Im. 33, 1341-1350.

Vignon-Clementel, I., Figueroa, C., Jansen, K., Taylor, C., 2010a. Outflow boundary conditions for 3D simulations of non-periodic blood flow and pressure fields in deformable arteries. Comp. Meth. Biomech. Biomed. Engnr. 111, 502-513.

Vignon-Clementel, I.E., Marsden, A.L., Feinstein, J.A., 2010b. A primer on computational simulation in congenital heart disease for the clinician. Progr. Ped. Card. 30, 3-13.

Volkwein, S., 1999. Proper orthogonal decomposition and singular value decomposition. Technical Report. Karl-Franzens-Univ. Graz \& Techn. Univ. Graz. Http://www.unigraz.at/imawww/volkwein/POD.pdf.

Wang, Z., Akhtar, I., Borggaard, J., Iliescu, T., 2012. Proper orthogonal decomposition closure models for turbulent flows: a numerical comparison. Computer Methods in Applied Mechanics and Engineering .

Yang, W., Vignon-Clementel, I.E., Troianowski, G., Reddy, V.M., Feinstein, J.A., Marsden, A.L., 2011. Hepatic blood flow distribution and performance in conventional and novel Y-graft Fontan geometries: A case series computational fluid dynamics study. J. Thorac. Cardiovasc. Surg. . 
Yano, M., PATERA, A.T., URBAN, K., 2012. A space-time certified reduced basis method for burgers' equation. Math. Mod. Meth. Appl. S.,(submitted) .

Yeung, J.J., Kim, H.J., Abbruzzese, T.A., Vignon-Clementel, I.E., Draney-Blomme, M.T., Yeung, K.K., Perkash, I., Herfkens, R.J., Taylor, C.A., Dalman, R.L., 2006. Aortoiliac hemodynamic and morphologic adaptation to chronic spinal cord injury. J. Vasc. Surg. 44, 1254-1265. 